\title{
Statistical signatures of multimode single-photon-added and -subtracted states of light
}

\author{
Mattia Walschaers, Claude Fabre, Valentina Parigi, and Nicolas Treps \\ Laboratoire Kastler Brossel, UPMC, Sorbonne Universités, CNRS, ENS PSL Research University, Collège de France, \\ CNRS, 4 Place Jussieu, 75252 Paris, France
}

(Received 29 August 2017; published 14 November 2017)

\begin{abstract}
The addition or subtraction of a photon from a Gaussian state of light is a versatile and experimentally feasible procedure to create non-Gaussian states. In multimode setups, these states manifest a wide range of phenomena when the photon is added or subtracted in a mode-tunable way. In this paper we derive the truncated correlations, which are multimode generalizations of cumulants, between quadratures in different modes as statistical signatures of these states. These correlations are then used to obtain the full multimode Wigner function, the properties of which are subsequently studied. In particular we investigate the effect of impurity in the subtraction or addition process and evaluate its impact on the negativity of the Wigner function. Finally, we elaborate on the generation of inherent entanglement through subtraction or addition of a photon from a pure squeezed vacuum.
\end{abstract}

DOI: 10.1103/PhysRevA.96.053835

\section{INTRODUCTION}

Continuous-variable (CV) quantum optics has ample advantages for quantum information processing. The most notable strength of general optical systems is their resilience against decoherence, which proves useful for quantum protocols. In CV quantum optics, states with arbitrarily many entangled modes (normalized solutions to Maxwell's equations) can be deterministically generated [1,2]. However, these experimentally generated states are Gaussian, i.e., they can be described by a multivariate Gaussian probability distribution on the optical phase space (for formal details, see Sec. II). Because Gaussian statistics can easily be simulated with classical computation resources [3], the use of these states in quantum computation is limited.

To reach full universal quantum computation, CV setups require at least one non-Gaussian ingredient. There have been several theoretical and experimental proposals to achieve this, ranging from ancillary Gottesman-Kitaev-Preskill states [4] to specific non-Gaussian gates, e.g., [5]. Within this paper we focus on photon addition and subtraction as de-Gaussification techniques [6]. Such techniques have, for example, proven their worth for entanglement distillation [7-9]. In particular, the subtraction of a photon is in essence a simple procedure that, as originally proposed [10], only requires a beam splitter and a photodetector. However, because beam splitters are not mode selective, this simple photon-subtraction scheme will increase the impurity of the state. To avoid such incoherent mixing of modes, a theory for coherent mode-selective photon subtraction was recently developed [11,12]. Considerable steps have already been undertaken to implement this coherent mode-dependent photon subtraction in a quantum frequency comb [13].

In this paper we extend the theoretical framework for such multimode photon-added and -subtracted states. Our central achievement is the derivation of the general Wigner function $[14,15]$ for these states. Even though the Wigner functions for (multi)photon-added and -subtracted states are known in

\footnotetext{
*mattia.walschaers@1kb.upmc.fr
}

several specific setups, e.g., [10,16-19], general multimode results are still lacking, even in the case of single-photon addition and subtraction.

We approach this problem from a statistical-mechanics perspective, by deriving truncated correlation functions [20-23] for these particular cases of mode-selective photon addition and subtraction from multimode Gaussian states. Truncated correlations as such are useful witnesses for the Gaussianity of states, but they are also connected to phasespace representations. Specifically, we employ the truncated correlations to derive the characteristic function, which upon Fourier transformation gives us the Wigner function; this key result is shown in (50). In the remainder of the work, we investigate the negativity of this Wigner function, which is an important indicator of the nonclassicality of the state [24-28] from a quantum probability theory perspective. Finally, we also investigate the entanglement properties that can be deduced from the Wigner function, which are ultimately the features that we want to exploit in future application in quantum technologies. This work elaborates on the details behind [29] and generalizes the results to nonpure photon addition and subtraction.

The paper is structured in three major parts. In Sec. II we introduce the mathematical formalism and concepts that mix techniques from quantum statistical mechanics [21-23] and quantum optics [14,15]. These techniques are applied in Sec. III to investigate multimode mode-selective photon addition and subtraction. To make our abstract results more concrete, we finally study two examples in Sec. IV: the subtraction and addition from the two-mode symmetrically squeezed vacuum and from an experimentally obtained state. The latter is an extension of the results of [29].

\section{MULTIMODE QUANTUM OPTICS ON PHASE SPACE}

\section{A. Optical phase space and quadrature operators}

The study of continuous-variable multimode quantum optics is in essence a study of quantum physics in a highdimensional phase space. For optical systems, the relevant phase space is generated by the real and imaginary parts 
of the contributing electric fields, the amplitude and phase quadratures, respectively.

The modal structure of light is essential in the present work. A mode is simply a normalized ${ }^{1}$ solution $u(\mathbf{r}, t)$ to Maxwell's equation, which has both a spatial and a temporal structure, as indicated by the arguments $\mathbf{r}$ and $t$, respectively. A general complex electric field $E(\mathbf{r}, t)$ can then be represented in terms of a mode basis $\left\{u_{j}(\mathbf{r}, t) \mid j=1, \ldots, m\right\}$ as

$$
E(\mathbf{r}, t)=\sum_{j=1}^{m}\left(x_{j}+i p_{j}\right) u_{j}(\mathbf{r}, t),
$$

where $x_{j}$ and $p_{j}$ are the amplitude and phase quadratures, respectively. Therefore, every vector $f=\left(x_{1}, \ldots, x_{m}\right.$, $\left.p_{1}, \ldots, p_{m}\right)^{t} \in \mathbb{R}^{2 m}$ can be associated with a set of phase and amplitude quadratures in the specific mode basis. The vector space $\mathbb{R}^{2 m}$ that is generated in this way is the optical phase space. As such, any vector $f \in \mathbb{R}^{2 m}$ represents a classical electromagnetic field. When, in addition, $f$ is normalized, this classical electric field is associated with a new mode. Thus, it is useful to introduce

$$
\mathcal{N}\left(\mathbb{R}^{2 m}\right)=\left\{f \in \mathbb{R}^{2 m} \mid\|f\|=1\right\}
$$

to describe modes. However, the dimension of $\mathcal{N}\left(\mathbb{R}^{2 m}\right)$ is larger than the number of modes $m$. This is a consequence of the complex amplitude of the field, which associates two quadratures with every mode. To faithfully reproduce the properties of these complex amplitudes in (1), the phase and amplitude quadratures are connected through a symplectic structure represented by a matrix $J$ that acts on the optical phase space $\mathbb{R}^{2 m}$ and has the properties

$$
\begin{aligned}
& J^{2}=-\mathbb{1}, \\
& \left(f_{1}, J f_{2}\right)=-\left(f_{2}, J f_{1}\right) \text { for all } f_{1}, f_{2} \in \mathbb{R}^{2 m},
\end{aligned}
$$

where $(\cdot, \cdot)$ denotes the standard inner product on $\mathbb{R}^{2 m}$. This structure implies that the optical phase space is a phase space as also studied in analytical mechanics. An important consequence of (4) is that $(f, J f)=0$ for every $f \in \mathbb{R}^{2 m}$. The orthogonal vectors $f, J f \in \mathcal{N}\left(\mathbb{R}^{2 m}\right)$ are associated with the same mode $u_{f}(\mathbf{r}, t)$ such that the space generated by $f$ and $J f$ is the two-dimensional phase space that describes all possible electromagnetic fields in mode $u_{f}(\mathbf{r}, t)$.

One can always construct an orthonormal symplectic basis $\mathcal{E}_{s}=\left\{e^{(1)}, \ldots, e^{(m)}, J e^{(1)}, \ldots, J e^{(m)}\right\}$ of the optical phase space, where $e^{(i)}$ is the basis vector that generates the phase-space axis that denotes the amplitude quadrature of mode $u_{i}(\mathbf{r}, t)$, whereas $J e^{(i)}$ generates the associated phase quadrature. The symplectic basis $\mathcal{E}_{s}$ of the optical phase space is directly associated with a mode basis $\left\{u_{j}(\mathbf{r}, t) \mid j=\right.$ $1, \ldots, m\}$. Hence, a change of basis in the optical phase space implies a change in mode basis.

When we combine the above optical phase space with the framework of statistical mechanics, we can describe classical

\footnotetext{
${ }^{1}$ The modes $u_{j}(\mathbf{r}, t)$ are functions in space and time, but are typically normalized only in the spatial degrees of freedom, i.e., $\int\left|u_{j}(\mathbf{r}, t)\right|^{2} d^{3} \mathbf{r}=1$ for any time $t$. For concrete examples and a more thorough introduction, see [30-32].
}

optics setups. However, to treat problems in multimode quantum optics, we must go through the procedure of canonical quantisation. To do so, we associate a quadrature operator $Q(f)$ with each $f \in \mathcal{N}\left(\mathbb{R}^{2 m}\right)$. These operators fulfill the crucial mathematical property

$$
Q\left(a f_{1}+b f_{2}\right)=a Q\left(f_{1}\right)+b Q\left(f_{2}\right)
$$

for all $f_{1}, f_{2} \in \mathcal{N}\left(\mathbb{R}^{2 m}\right)$ and $a, b \in \mathbb{R}$ with $a^{2}+b^{2}=1$. This property implies that the operator $Q(f)$ is independent of the basis chosen to express $f$. Moreover, these operators are governed by the canonical commutation relation $[21,33]$

$$
\left[Q\left(f_{1}\right), Q\left(f_{2}\right)\right]=-2 i\left(f_{1}, J f_{2}\right) \quad \text { for all } f_{1}, f_{2} \in \mathcal{N}\left(\mathbb{R}^{2 m}\right) \text {. }
$$

We have defined (6) such that the operator $Q(f)$ corresponds to a quadrature operator with the shot noise equal to one.

The linearity condition (5) can be extended to all $a, b \in \mathbb{R}$ to define operators $Q(\alpha)$ for non-normalized $\alpha \in \mathbb{R}^{2 m}$. This generalization does not lead to any mathematical problems and (6) still holds. Physically, such different norms of $\alpha$ can be associated with rescaled quadrature measurements. In this article, the generalized quadratures will be used to limit notational overhead in the definition of the displacement operator (7).

\section{B. Representing quantum states}

Because quantum physics is a statistical theory, we require a mathematical object to describe the statistics of measurements: the quantum state. We focus on systems that can accurately be represented in a Hilbert space $\mathcal{H}$, on which $Q(f)$ is an (unbounded) operator. The quantum state can then be represented by a density operator $\rho$, which is positive and has $\operatorname{tr} \rho=1 .^{2}$

However, the density operator is not the most practical tool to characterize a state of a continuous-variable system. Quasiprobability distributions on phase space are a common and practical alternative, not only due to their importance to interpret the fundamental physics of the state, but also because they can be measured experimentally. Throughout this paper we will particularly emphasize the Wigner function as an important tool, because, at least for small mode numbers, it can be experimentally reconstructed through tomographic methods.

From the mathematical point of view, we start by constructing the characteristic function in order to derive the Wigner function. To do so, we first define the displacement operator

$$
D(\alpha) \equiv \exp [-i Q(J \alpha) / 2], \quad \alpha \in \mathbb{R}^{2 m} .
$$

Importantly, $\alpha$ is generally not normalized as its norm dictates the distance of the displacement. Indeed, this operator's action on a quadrature operator is given by

$$
D(-\alpha) Q(f) D(\alpha)=Q(f)+(f, \alpha)
$$

\footnotetext{
${ }^{2}$ We may, in principle, also apply the methods in the present work to more general states and their representation of the $C^{*}$-algebra of the canonical commutation relations. This allows us to treat systems of infinitely many modes.
} 
such that the strength and direction of the displacement are given by $\|\alpha\|$ and $\alpha /\|\alpha\|$, respectively. Via the displacement operator, we can introduce the characteristic function as

$$
\chi(\alpha) \equiv \operatorname{tr}\{\rho D(2 J \alpha)\}=\operatorname{tr}\{\rho \exp [i Q(\alpha)]\} .
$$

Subsequently, one can construct the Wigner function by a multidimensional Fourier transformation

$$
W(\beta)=\frac{1}{(2 \pi)^{2 m}} \int_{\mathbb{R}^{2 m}} d \alpha \chi(\alpha) e^{-i(\alpha, \beta)} \quad \text { for } \beta \in \mathbb{R}^{2 m},
$$

where $\beta$ indicates a point in phase space, hence it need not be normalized. The Wigner function shares the normalization properties of a probability distribution and its marginals are probability measures. However, the full Wigner function is merely a quasiprobability distribution in the sense that it can assume negative values for some regions of phase space. It is this negativity that sets quantum mechanics apart from classical probabilistic theories on phase space. As such, negativity can be seen as a genuine sign of quantumness, which was also associated with quantum supremacy [3,28].

Furthermore, the characteristic function can be directly linked to the cumulants of a specific quadrature measurement statistics. Indeed, $\ln \chi(\alpha)$ is also known as the cumulantgenerating function, which implies that

$$
\left.\frac{\partial^{n}}{\partial \lambda^{n}} \ln \chi(\lambda f)\right|_{\lambda=0} \equiv\left\langle Q(f)^{n}\right\rangle_{T},
$$

with $\lambda \in \mathbb{R}$ and $f \in \mathcal{N}\left(\mathbb{R}^{2 m}\right)$. Here $\left\langle Q(f)^{n}\right\rangle_{T}$ denotes the $n$th cumulant for the measurement of the quadrature $Q(f)$. A straightforward calculation [21,23] now shows that one can recast the characteristic function in the form

$$
\chi(\alpha)=\exp \left\{\sum_{n=1}^{\infty} \frac{i^{n}}{n !}\left\langle Q(\alpha)^{n}\right\rangle_{T}\right\}, \quad \alpha \in \mathbb{R}^{2 m} .
$$

Thus, knowledge of all the cumulants for all the different quadratures, i.e., for all $f \in \mathcal{N}\left(\mathbb{R}^{2 m}\right)$ and all orders $n$, implies full knowledge of the quantum state.

We now introduce these cumulants in a more explicit form, by treating them as a special case of truncated correlation functions.

\section{Truncated correlation functions}

The cumulants of $Q(f)$ (11) are related to the statistics of a single mode $f \in \mathcal{N}\left(\mathbb{R}^{2 m}\right)$ and do not explicitly elucidate how different modes are correlated. However, to study such questions the cumulant can be generalized to a multimode form that is commonly referred to as the truncated correlation [20] between different quadratures. ${ }^{3}$

Truncated correlation functions are the multivariate extensions of cumulants and describe how quadratures for different modes are correlated. They, too, are generated using displacement operators $D(\alpha)$ [Eq. (7)]. In general,

\footnotetext{
${ }^{3}$ In statistics literature one may also encounter the terminology "joint cumulant," whereas in quantum field theory one also refers to the "connected part of the correlation."
}

we obtain

$$
\begin{aligned}
& \left\langle Q\left(f_{1}\right) \cdots Q\left(f_{n}\right)\right\rangle_{T} \\
& \left.\quad \equiv \frac{\partial \ln \operatorname{tr}\left[\rho D\left(2 \lambda_{1} J f_{1}\right) \ldots D\left(2 \lambda_{n} J f_{n}\right)\right]}{\partial \lambda_{1} \cdots \partial \lambda_{n}}\right|_{\lambda_{1}=\cdots=\lambda_{n}=0},
\end{aligned}
$$

which can be related to $\chi(\alpha)$ in (9) through the identity $D(\alpha) D(\beta)=D(\alpha+\beta) \exp \{-i(\alpha, J \beta) / 4\}$.

In an experimental setting, it is more practical to obtain the truncated correlation functions by jointly measuring distinct quadratures and following a recursive recipe

$$
\begin{aligned}
\left\langle Q\left(f_{1}\right)\right\rangle_{T}= & \operatorname{tr}\left\{\rho Q\left(f_{1}\right)\right\}, \\
\left\langle Q\left(f_{1}\right) Q\left(f_{2}\right)\right\rangle_{T}= & \operatorname{tr}\left\{\rho Q\left(f_{1}\right) Q\left(f_{2}\right)\right\} \\
& -\left\langle Q\left(f_{1}\right)\right\rangle_{T}\left\langle Q\left(f_{2}\right)\right\rangle_{T}, \\
\left\langle Q\left(f_{1}\right) Q\left(f_{2}\right) Q\left(f_{3}\right)\right\rangle_{T}= & \operatorname{tr}\left\{\rho Q\left(f_{1}\right) Q\left(f_{2}\right) Q\left(f_{3}\right)\right\} \\
& -\left\langle Q\left(f_{1}\right) Q\left(f_{2}\right)\right\rangle_{T}\left\langle Q\left(f_{3}\right)\right\rangle_{T} \\
& -\left\langle Q\left(f_{1}\right) Q\left(f_{3}\right)\right\rangle_{T}\left\langle Q\left(f_{2}\right)\right\rangle_{T} \\
& -\left\langle Q\left(f_{2}\right) Q\left(f_{3}\right)\right\rangle_{T}\left\langle Q\left(f_{1}\right)\right\rangle_{T} \\
& -\left\langle Q\left(f_{1}\right)\right\rangle_{T}\left\langle Q\left(f_{3}\right)\right\rangle_{T}\left\langle Q\left(f_{2}\right)\right\rangle_{T},
\end{aligned}
$$

These truncated correlation functions are experimentally measurable through, for example, multimode homodyne measurement [34-36]. By expanding $\alpha \in \mathbb{R}^{2 m}$ in a specific mode basis in (12), the role of truncated correlations becomes apparent. Thus, the set of truncated correlations forms an important tool for characterization. More specifically, one may wonder to what order one needs to measure these correlations to extract a given property of the state. Such a property, which is of special interest throughout this text, is the state's Gaussianity.

\section{Gaussian states}

Of particular importance in quantum optics are the Gaussian states. In the broad sense, a state of a CV system is said to be Gaussian if it induces Gaussian statistics in all modes. This implies that the function $\chi$, and hence also the Wigner function, is a multivariate Gaussian $[14,15]$

$$
\begin{aligned}
\chi_{G}(\alpha) & =\exp \left\{-\frac{(\alpha, V \alpha)}{2}+i(\xi, \alpha)\right\}, \\
W_{G}(\beta) & =\frac{\exp \left\{-\frac{1}{2}\left((\beta-\xi), V^{-1}(\beta-\xi)\right)\right\}}{(2 \pi)^{m} \sqrt{\operatorname{det} V}},
\end{aligned}
$$

where $\xi$ is a vector that describes the states displacement and $V$ is referred to as the covariance matrix. Therefore, $V$ is a positive-semidefinite matrix on $\mathbb{R}^{2 m}$, which describes the correlations between different field quadratures in a specific mode basis. A crucial demand for this $V$ to be associated with a well-defined quantum state is given by [21]

$$
\left(f_{1}, V f_{1}\right)\left(f_{2}, V f_{2}\right) \geqslant\left|\left(f_{1}, J f_{2}\right)\right|^{2} \text { for all } f_{1}, f_{2} \in \mathcal{N}\left(\mathbb{R}^{2 m}\right) \text {, }
$$


which is the multimode version of Heisenberg's uncertainty relation. Alternatively, the properties of $V$ can also be expressed by the condition $V+i J \geqslant 0$.

The insertion of (15) in (13) imposes important conditions upon the truncated correlations of Gaussian states. At first it is directly obtained that

$$
\begin{gathered}
\langle Q(f)\rangle_{T}=(\xi, f), \\
\left\langle Q\left(f_{1}\right) Q\left(f_{2}\right)\right\rangle_{T}=\left(f_{1}, V f_{2}\right)-i\left(f_{1}, J f_{2}\right) .
\end{gathered}
$$

Furthermore, we deduce the general condition that for a Gaussian state

$$
\left\langle Q\left(f_{1}\right) \cdots Q\left(f_{n}\right)\right\rangle_{T}=0, \quad n>2,
$$

for all $f_{1}, \ldots, f_{n} \in \mathcal{N}\left(\mathbb{R}^{2 m}\right)$. The implication of (20) is that all non-Gaussian states must have nonzero truncated correlations. Furthermore, it was shown [37] that for non-Gaussian states, there is never an order from which onward the truncated correlation functions become zero. Therefore, these functions are ideal tools for the operational characterisation of nonGaussian states. Specifically, in multimode systems where full tomographies tend to be completely unfeasible, they are an experimentally accessible alternative.

\section{E. Entanglement}

In the context of quantum physics, one often associates correlations with the study of entanglement, which is commonly seen as an important resource for quantum computation and quantum communication. The profound advantage of $\mathrm{CV}$ quantum optics is the simplicity with which Gaussian entanglement between modes can be generated. Continuousvariable entanglement is strongly dependent of the mode basis in which the problem is described. To see this, it is instructive to consider an arbitrary symplectic basis $\mathcal{B}=$ $\left\{b^{(1)}, \ldots, b^{(m)}, J b^{(1)}, \ldots, J b^{(m)}\right\}$ of $\mathbb{R}^{2 m}$ and express $\beta \in \mathbb{R}^{2 m}$ in (10) in this basis:

$$
\beta=\sum_{i=1}^{m} \zeta_{x}^{(i)} b^{(i)}+\zeta_{p}^{(i)} J b^{(i)}
$$

In this mode basis, the Wigner function is a function of the $\zeta$ variables, i.e., $W(\beta)=W\left(\zeta_{x}^{(1)}, \ldots, \zeta_{x}^{(m)}, \zeta_{p}^{(1)}, \ldots, \zeta_{p}^{(m)}\right){ }^{4}$

We refer to a CV state as fully separable in the mode basis $\mathcal{B}$ when its Wigner function can be written as

$$
\begin{aligned}
W & \left(\zeta_{x}^{(1)}, \ldots, \zeta_{x}^{(m)}, \zeta_{p}^{(1)}, \ldots, \zeta_{p}^{(m)}\right) \\
& =\int d \lambda p(\lambda) \prod_{i=1}^{m} W_{\lambda}\left(\zeta_{x}^{(i)}, \zeta_{p}^{(i)}\right),
\end{aligned}
$$

a statistical mixture of a product of single-mode Wigner functions. To obtain a statistical mixture, $\lambda$ must correspond to a way of labeling states and $p(\lambda)$ is a probability distribution on this set of labels. Any state for which (22) does not hold is said

\footnotetext{
${ }^{4} \mathrm{~A}$ different choice of mode basis $\mathcal{B}$ changes the form of the Wigner function. Thus, physical properties such as entanglement, which depend on the form of the Wigner function, depend on the chosen mode basis.
}

to be entangled in mode basis $\mathcal{B}$. Note that one can introduce more refined terminology for multimode entanglement [38]. Analyzing such different types of multipartite entanglement, however, falls beyond the scope of this work.

It is natural to ask whether there always exists a mode basis in which the state is separable. We will provide a negative answer to this question by showing that this is generally not the case for photon-added and -subtracted states. If we can construct a mode basis for which (22) holds, we will refer to the state as passively separable, to highlight that any entanglement present in a specific mode basis can be undone by passive linear optics. States that are not passively separable are now referred to as inherently entangled.

We show now that the Gaussian states of Sec. IID are always passively separable, by using the properties of their covariance matrices. The Wigner function (16) of a nondisplaced (i.e., $\xi=0$ ) Gaussian state $\rho_{G}$ is completely governed by the positive-semidefinite covariance matrix $V$, which can be decomposed as $V=S^{t} \Delta S$ through the Williamson decomposition. Here $S$ is a symplectic matrix and $\Delta \geqslant \mathbb{1}$ is diagonal (the diagonal elements of $\Delta$ are known as the symplectic spectrum). Because $S$ is symplectic, it can be further decomposed with the Bloch-Messiah decomposition: $S=O^{\prime} K O$, where $O$ and $O^{\prime}$ are orthogonal and symplectic and $K$ is diagonal and symplectic. This now allows us to rewrite $V=O^{t} K V_{\text {th }} K O$, where $V_{\text {th }}=O^{\prime t} \Delta O^{\prime} \geqslant \mathbb{1}$ is the covariance matrix of a thermal state. We use this structure to separate the covariance into a pure part and added classical noise

$$
V=V_{s}+V_{c} .
$$

Here $V_{s}=O^{t} K^{2} O$ is the covariance matrix of a pure squeezed vacuum state $\rho_{s}$ and $V_{c}=O^{t} K\left(V_{\text {th }}-\mathbb{1}\right) K O$ is the covariance matrix of the additional noise. Note that, a priori, $V_{c}$ does not fulfill (17) and is therefore not the covariance matrix of a quantum state. We can think of the state characterized by $V$ as being generated by injecting $\rho_{s}$ into a noisy Gaussian channel $[39,40]$. We obtain that

$$
\rho_{G}=\int_{\mathbb{R}^{2 m}} d^{2 m} \xi^{\prime} D\left(\xi^{\prime}\right) \rho_{s} D\left(-\xi^{\prime}\right) \frac{\exp \left\{-\frac{\left(\xi, V_{c}^{-1} \xi\right)}{2}\right\}}{(2 \pi)^{m} \sqrt{\operatorname{det} V_{c}}},
$$

which implies that the Wigner function for $\rho_{G}$ can be represented by

$$
W_{G}(\beta)=\int d^{2 m} \xi W_{s}(\beta-\xi) p_{c}(\xi),
$$

where

$$
p_{c}(\xi)=\frac{\exp \left\{-\frac{\left(\xi, V_{c}^{-1} \xi\right)}{2}\right\}}{(2 \pi)^{m} \sqrt{\operatorname{det} V_{c}}}
$$

and

$$
W_{s}(\beta)=\frac{\exp \left\{-\frac{1}{2}\left(\beta, V_{s}^{-1} \beta\right)\right\}}{(2 \pi)^{m} \sqrt{\operatorname{det} V_{s}}} .
$$

The Bloch-Messiah decomposition naturally gives a specific mode basis (obtained through the orthogonal transformation $O$ ) in which $W_{s}$ factorizes for any $\xi \in \mathbb{R}^{2 m}$. In this mode basis, the Wigner function (25) has the form (22), which implies that the state $\rho_{G}$ is passively separable. Thus, any entanglement 
that is present in the original mode basis can be undone by a passive linear optics circuit that is described by $O^{t}$ or by measuring quadratures in mode basis associated with $O$.

Thus we provided an explicit construction of a linear optics operation to render a given Gaussian state separable. For mixed states this is typically not the only linear optics operation that can undo entanglement. Indeed, the core ingredient of the decomposition (24) is (23) such that for every pure-state covariance matrix $V_{s}^{\prime} \leqslant V$, we can set $V_{c}=V-V_{s}^{\prime}$ in (23). Because $V_{s}^{\prime}$ characterizes a pure Gaussian state, we can find a mode basis of symplectic eigenvectors of $V_{s}^{\prime}$. We can thus simply diagonalize $V_{s}^{\prime}=O^{\prime t} K^{\prime 2} O^{\prime}$, where $O^{\prime t}$ describes an alternative linear optics circuit that can undo entanglement in the Gaussian state. The above method, using Williamson and Bloch-Messiah decomposition, shows that such a $V_{s}^{\prime}$ always exists.

For simplicity, we assumed that $\rho_{G}$ was nondisplaced. However, the argument is straightforwardly extended to displaced Gaussian states by letting the displacement operator act on $\rho_{G}$.

\section{SINGLE-PHOTON-ADDED AND -SUBTRACTED GAUSSIAN STATES}

\section{A. Induced correlations}

As we argued in the Introduction, non-Gaussianity is a crucial ingredient to achieve universal quantum computation. Moreover, for technological applications, it is essential that the complexity of any quantum device can be increased, hence requiring a sense of scalability. In $\mathrm{CV}$ quantum optics, we first and foremost consider such scalability in the number of modes. Therefore, we must consider a multimode setup, in which we can incorporate a non-Gaussian operation. From an experimental perspective, a promising procedure to fulfill these conditions is mode-selective photon subtraction [11-13] or addition $[6,41]$.

In this work we limit ourselves to the subtraction or addition of a single photon in a setup with an arbitrary mode number $m$. To effectively model the associated subtraction and addition procedures, we must introduce the annihilation and creation operators for an arbitrary vector in phase space $g \in \mathcal{N}\left(\mathbb{R}^{2 m}\right)$, $a(g)$ and $a^{\dagger}(g)$, respectively. In our framework, they are defined as

$$
\begin{aligned}
a^{\dagger}(g) & \equiv \frac{1}{2}[Q(g)-i Q(J g)], \\
a(g) & \equiv \frac{1}{2}[Q(g)+i Q(J g)],
\end{aligned}
$$

from which we directly obtain an alternative version of the canonical commutation relation (6),

$$
\left[a\left(g_{1}\right), a^{\dagger}\left(g_{2}\right)\right]=\left(g_{1}, g_{2}\right)+i\left(g_{1}, J g_{2}\right) .
$$

These operators create or annihilate photons in a specific mode, represented by $g$. However, $g$ is a vector in the $2 m$-dimensional phase space, whereas there are only $m$ modes. Therefore, we stress that $a^{\dagger}(J g)=-i a^{\dagger}(g)$ such that the photons created by the operators $a^{\dagger}(g)$ and $a^{\dagger}(J g)$ clearly only differ by a global phase. As global phases have no physical importance in quantum physics, the creation operators $a^{\dagger}(g)$ and $a^{\dagger}(J g)$ really create a photon in the same mode.

We now focus on an arbitrary nondisplaced Gaussian state, which we formally describe by a density matrix $\rho_{G}$, and convert it to a non-Gaussian state by means of mode-selective photon addition or subtraction in a mode $g \in \mathcal{N}\left(\mathbb{R}^{2 m}\right)$. As was argued in Sec. II D, this nondisplaced Gaussian state can be completely characterized by its covariance matrix $V$. The new photon-added and -subtracted states' density operators are then given by

$$
\rho_{-}=\frac{a(g) \rho_{G} a^{\dagger}(g)}{\langle\hat{n}(g)\rangle_{G}}
$$

for subtraction and

$$
\rho_{+}=\frac{a^{\dagger}(g) \rho_{G} a(g)}{\langle\hat{n}(g)\rangle_{G}+1}
$$

for addition. We introduced the notation $\langle\cdot\rangle_{G} \equiv \operatorname{tr}\left(\rho_{G} \cdot\right)$ for the expectation values in the state $\rho_{G}$ and $\hat{n}(g) \equiv a^{\dagger}(g) a(g)$ for the number operator that counts the number of photons in the mode $g \in \mathcal{N}\left(\mathbb{R}^{2 m}\right)$.

In order to characterize the non-Gaussian features of the system, we follow the ideas of Sec. IIC and evaluate the truncated correlation functions. If the state is indeed nonGaussian, we should obtain nonzero values for the truncated correlation functions of some order beyond than 2. However, because we intend to use the recursive procedure of Sec. II C to evaluate the correlations, it is instructive to start by evaluating the two-point correlation $\left\langle Q\left(f_{1}\right) Q\left(f_{2}\right)\right\rangle_{T}$ for arbitrary $f_{1}, f_{2} \in$ $\mathcal{N}\left(\mathbb{R}^{2 m}\right)$. Because the state is nondisplaced, by definition $\langle Q(f)\rangle_{T}=0$ and we obtain that for the photon-subtracted (hence the minus sign superscript) state

$$
\begin{aligned}
\left\langle Q\left(f_{1}\right) Q\left(f_{2}\right)\right\rangle_{T}^{-} & =\operatorname{tr}\left\{\rho_{-} Q\left(f_{1}\right) Q\left(f_{2}\right)\right\} \\
& =\frac{\left\langle a^{\dagger}(g) Q\left(f_{1}\right) Q\left(f_{2}\right) a(g)\right\rangle_{G}}{\langle\hat{n}(g)\rangle_{G}},
\end{aligned}
$$

where we used (30) and the cyclic property of the trace. Analogously, for photon addition we obtain

$$
\left\langle Q\left(f_{1}\right) Q\left(f_{2}\right)\right\rangle_{T}^{+}=\frac{\left\langle a(g) Q\left(f_{1}\right) Q\left(f_{2}\right) a^{\dagger}(g)\right\rangle_{G}}{\langle\hat{n}(g)\rangle_{G}+1} .
$$

The property (20) for nondisplaced Gaussian states implies that expectation values of products of quadrature operators factorize in pairs [20,21,23,42]. Combining this with the definition (28) for the creation and annihilation operators in terms of quadratures, and with the linearity of the trace, we find

$$
\begin{aligned}
\left\langle Q\left(f_{1}\right) Q\left(f_{2}\right)\right\rangle_{T}^{-}= & \frac{\left\langle a^{\dagger}(g) Q\left(f_{1}\right)\right\rangle_{G}\left\langle Q\left(f_{2}\right) a(g)\right\rangle_{G}}{\langle\hat{n}(g)\rangle_{G}} \\
& +\frac{\left\langle a^{\dagger}(g) Q\left(f_{2}\right)\right\rangle_{G}\left\langle Q\left(f_{1}\right) a(g)\right\rangle_{G}}{\langle\hat{n}(g)\rangle_{G}} \\
& +\left\langle Q\left(f_{1}\right) Q\left(f_{2}\right)\right\rangle_{G}
\end{aligned}
$$

and

$$
\begin{aligned}
\left\langle Q\left(f_{1}\right) Q\left(f_{2}\right)\right\rangle_{T}^{+}= & \frac{\left\langle a(g) Q\left(f_{1}\right)\right\rangle_{G}\left\langle Q\left(f_{2}\right) a^{\dagger}(g)\right\rangle_{G}}{\langle\hat{n}(g)\rangle_{G}+1} \\
& +\frac{\left\langle a(g) Q\left(f_{2}\right)\right\rangle_{G}\left\langle Q\left(f_{1}\right) a^{\dagger}(g)\right\rangle_{G}}{\langle\hat{n}(g)\rangle_{G}+1} \\
& +\left\langle Q\left(f_{1}\right) Q\left(f_{2}\right)\right\rangle_{G} .
\end{aligned}
$$


To proceed with the evaluation, we use (19) and (28) to obtain

$$
\begin{aligned}
\left\langle a^{\dagger}(g) Q(f)\right\rangle_{G} & =\frac{1}{2}\{(f,[V-\mathbb{1}] g)-i(f,[V-\mathbb{1}] J g)\} \\
\left\langle Q(f) a^{\dagger}(g)\right\rangle_{G} & =\frac{1}{2}\{(f,[V+\mathbb{1}] g)-i(f,[V+\mathbb{1}] J g)\} \\
\langle Q(f) a(g)\rangle_{G} & =\frac{1}{2}\{(f,[V-\mathbb{1}] g)+i(f,[V-\mathbb{1}] J g)\} \\
\langle a(g) Q(f)\rangle_{G} & =\frac{1}{2}\{(f,[V+\mathbb{1}] g)+i[(f,[V+\mathbb{1}] J g)]\} \\
\langle\hat{n}(g)\rangle_{G} & =\frac{1}{4}\{(g, V g)+(J g, V J g)-2\} .
\end{aligned}
$$

When we insert these results in (35), we ultimately obtain that for the photon-subtracted nondisplaced Gaussian state

$$
\left\langle Q\left(f_{1}\right) Q\left(f_{2}\right)\right\rangle_{T}^{ \pm}=\left\langle Q\left(f_{1}\right) Q\left(f_{2}\right)\right\rangle_{G}+\left(f_{1}, A_{g}^{ \pm} f_{2}\right),
$$

with

$$
\begin{aligned}
\left(f_{1}, A_{g}^{-} f_{2}\right) \equiv & \frac{\left\langle a^{\dagger}(g) Q\left(f_{1}\right)\right\rangle_{G}\left\langle Q\left(f_{2}\right) a(g)\right\rangle_{G}}{\langle\hat{n}(g)\rangle_{G}} \\
& +\frac{\left\langle a^{\dagger}(g) Q\left(f_{2}\right)\right\rangle_{G}\left\langle Q\left(f_{1}\right) a(g)\right\rangle_{G}}{\langle\hat{n}(g)\rangle_{G}}
\end{aligned}
$$

and

$$
\begin{aligned}
\left(f_{1}, A_{g}^{+} f_{2}\right) \equiv & \frac{\left\langle a(g) Q\left(f_{1}\right)\right\rangle_{G}\left\langle Q\left(f_{2}\right) a^{\dagger}(g)\right\rangle_{G}}{\langle\hat{n}(g)\rangle_{G}+1} \\
& +\frac{\left\langle a(g) Q\left(f_{2}\right)\right\rangle_{G}\left\langle Q\left(f_{1}\right) a^{\dagger}(g)\right\rangle_{G}}{\langle\hat{n}(g)\rangle_{G}+1},
\end{aligned}
$$

where $A_{g}^{ \pm}$is a matrix that acts on the space $\mathbb{R}^{2 m}$. Inserting (37) in (38) directly leads to

$$
A_{g}^{ \pm}=2 \frac{(V \pm \mathbb{1})\left(P_{g}+P_{J g}\right)(V \pm \mathbb{1})}{\operatorname{tr}\left\{(V \pm \mathbb{1})\left(P_{g}+P_{J g}\right)\right\}}
$$

Here we introduced $P_{g}$ and $P_{J g}$ as the projectors on the vectors $g$ and $J g$, respectively. This implies that $P_{g}+P_{J g}$ is the projector on the two-dimensional phase space, associated with the mode in which the photon was subtracted. It can directly be verified that $A_{g}^{ \pm}$describes additional correlations between quadratures that are induced by the photon-subtraction or -addition process. Ultimately, these additional correlations are completely determined by the mode $g$ from which the photon is subtracted and the correlation matrix $V$ of the initial nondisplaced Gaussian state $\rho_{G}$.

Experimentally, however, it is hard to guarantee that (30) and (31) are the exact states that we obtain. In general, the subtraction process adds some degree of impurity. There are various sources of impurities in an experimental context, ranging from photon losses to contributions of higher photon numbers in the subtraction [13], which go beyond the scope of the present work. Nevertheless, we consider one important type of impurity in the subtraction process, related to the lack of control of the mode selectivity [12]. In the most extreme case, one may think of photon subtraction by means of a beam splitter, where it is impossible to infer from which mode the photon originated in the case of copropagating modes. In general terms, it is hard to control exactly in which mode the photon is added or subtracted [12]. This implies that we have to deal with a mixture of the form

$$
\rho_{-}=\frac{\sum_{k} \gamma_{k} a\left(g_{k}\right) \rho_{G} a^{\dagger}\left(g_{k}\right)}{\sum_{k} \gamma_{k}\left\langle\hat{n}\left(g_{k}\right)\right\rangle_{G}}
$$

with $\sum_{k} \gamma_{k}=1$ and $\gamma_{k} \geqslant 0$, for subtraction, or

$$
\rho_{+}=\frac{\sum_{k} \gamma_{k} a^{\dagger}\left(g_{k}\right) \rho_{G} a\left(g_{k}\right)}{1+\sum_{k} \gamma_{k}\left\langle\hat{n}\left(g_{k}\right)\right\rangle_{G}}
$$

with $\sum_{k} \gamma_{k}=1$ and $\gamma_{k} \geqslant 0$, for addition. The details of the participating modes and the $\gamma_{k}$ depend strongly on the experimental setup and can be estimated through a detailed modeling [11,12].

Through the linearity of the expectation value, we can directly verify that

$$
\left\langle Q\left(f_{1}\right) Q\left(f_{2}\right)\right\rangle_{T}^{ \pm}=\left\langle Q\left(f_{1}\right) Q\left(f_{2}\right)\right\rangle_{G}+\left(f_{1}, A_{\text {mix }}^{ \pm} f_{2}\right),
$$

where

$$
A_{\text {mix }}^{ \pm}=2(V \pm \mathbb{1}) \frac{\sum_{k} \gamma_{k}\left(P_{g_{k}}+P_{J g_{k}}\right)}{\operatorname{tr}\left\{(V \pm \mathbb{1}) \sum_{k} \gamma_{k}\left(P_{g_{k}}+P_{J g_{k}}\right)\right\}}(V \pm \mathbb{1}) .
$$

We use (42) to evaluate the higher-order truncated correlations in Appendix A. This leads to the remarkable result that these truncated correlations too are governed by the matrix $A_{\mathrm{mix}}^{ \pm}$. As a final result, we obtain

$$
\begin{aligned}
& \left\langle Q\left(f_{1}\right) \cdots Q\left(f_{2 k}\right)\right\rangle_{T} \\
& \quad=(-1)^{k-1}(k-1) ! \sum_{p \in \mathcal{P}^{(2)}} \prod_{i \in p}\left(f_{i_{1}}, A_{\text {mix }}^{ \pm} f_{i_{2}}\right), \\
& \left.Q\left(f_{1}\right) \cdots Q\left(f_{2 k-1}\right)\right\rangle_{T}=0
\end{aligned}
$$

for all $k>1$. Here $\mathcal{P}^{(2)}$ indicates the set of all pair partitions, i.e., all the ways of dividing the set $\left\{f_{1}, \ldots, f_{2 k}\right\}$ up in $k$ pairs. In the literature, e.g., [43], this partition is also known as a perfect matching. For even orders, the truncated correlations (44) are generally nonzero. This is a clear statistical signature of the non-Gaussian character of the state.

\section{B. Phase-space representations}

\section{Multimode Wigner function}

To highlight that the above truncated correlation functions grant us full knowledge of the quantum state, we use them to construct the quantum characteristic function for the photon-subtracted state (30) by virtue of (12). To do so, we need to know the state's cumulants, which are the truncated correlations (44) for $f_{1}=f_{2}=\cdots=f_{2 k}=f$. Central in this evaluation is that every pair partition $p \in \mathcal{P}^{(2)}$ in (44) contributes the same term $\left(f, A_{\text {mix }}^{ \pm} f\right)^{k}$ because the contribution of each mode is the same. We only need to count the number of pair partitions to know which combinatorial factor to add. We find that the cumulant is given by

$$
\left\langle Q(f)^{2 k}\right\rangle_{T}=(-1)^{k-1} \frac{(2 k-1) !}{2^{k-1}}\left(f, A_{\text {mix }}^{ \pm} f\right)^{k}+(f, V f) \delta_{k, 1},
$$

$$
\left\langle Q(f)^{2 k-1}\right\rangle_{T}=0
$$


It now remains to evaluate the series in Eq. (12), for which we find that

$$
\begin{aligned}
& \sum_{n=1}^{\infty} \frac{i^{n} \lambda^{n}}{n !}\left\langle Q(f)^{n}\right\rangle_{T} \\
& \quad=-\frac{\lambda^{2}(f, V f)}{2}+\sum_{k=1}^{\infty} \frac{i^{2 k} \lambda^{2 k}}{(2 k) !}(-1)^{k-1} \frac{(2 k-1) !}{2^{k-1}}\left(f, A_{\text {mix }}^{ \pm} f\right)^{k} \\
& \quad=-\frac{\lambda^{2}(f, V f)}{2}-\sum_{k=1}^{\infty} \frac{1}{k}\left(\frac{\lambda^{2}\left(f, A_{\text {mix }}^{ \pm} f\right)}{2}\right)^{k}
\end{aligned}
$$

where the series was already rewritten to only sum over the even cumulants since all odd contributions are zero. The final series in (47) is subtle because it does not necessarily converge. However, $\chi(\lambda f)$ maps the points of phase space to the complex plane; as such we may resort to an analytical continuation of the series to obtain that

$$
\begin{aligned}
\chi(\lambda f) & =\exp \left\{-\frac{\lambda^{2}}{2}(f, V f)-\sum_{k=1}^{\infty} \frac{1}{k}\left(\frac{\lambda^{2}\left(f, A_{\text {mix }}^{ \pm} f\right)}{2}\right)^{k}\right\} \\
& =\left(1-\frac{\lambda^{2}\left(f, A_{\text {mix }}^{ \pm} f\right)}{2}\right) \exp \left\{-\frac{\lambda^{2}}{2}(f, V f)\right\} .
\end{aligned}
$$

Therefore, we have obtained the quantum characteristic function (9) to fully characterize the states. In principle, this also allows us to derive the Wigner function by means of a multidimensional Fourier transformation. We may formally write the Wigner function as

$$
\begin{aligned}
W^{ \pm}(\beta)= & \frac{1}{(2 \pi)^{2 m}} \int_{\mathbb{R}^{2 m}} d \alpha \chi(\alpha) e^{-i(\alpha, \beta)} \\
= & \frac{1}{(2 \pi)^{2 m}} \int_{\mathbb{R}^{2 m}} d \alpha\left(1-\frac{\left(\alpha, A_{\text {mix }}^{ \pm} \alpha\right)}{2}\right) \\
& \times \exp \left\{-\frac{(\alpha, V \alpha)}{2}-i(\alpha, \beta)\right\} .
\end{aligned}
$$

This Fourier transform is explicitly computed in Appendix B and leads to

$$
W^{ \pm}(\beta)=\frac{1}{2}\left[\left(\beta, V^{-1} A_{\text {mix }}^{ \pm} V^{-1} \beta\right)-\operatorname{tr}\left(V^{-1} A_{\text {mix }}^{ \pm}\right)+2\right] W_{G}(\beta),
$$

where $W_{G}(\beta)$ is the Wigner function of the initial Gaussian state (16) before the addition or subtraction of the photon. This now gives us the full multimode Wigner functions of a nondisplaced photon-added or -subtracted state. We observe that the general structure of the Wigner function is given by a multivariate polynomial of order 2, multiplied by the Gaussian Wigner function of the initial state.

\section{Negativity}

The negativity of the Wigner function is often seen as a genuine quantum feature in CV systems. With (50) we have all the tools at hand to analyze such features in the Wigner function.

At first, we note that the Wigner function (50) is negative if and only if there are vectors $\beta \in \mathbb{R}^{2 m}$ for which

$$
\left(\beta, V^{-1} A_{\text {mix }}^{ \pm} V^{-1} \beta\right)-\operatorname{tr}\left(V^{-1} A_{\text {mix }}^{ \pm}\right)+2 \leqslant 0 .
$$

However, it is directly verified that $V^{-1} A_{\mathrm{mix}}^{ \pm} V^{-1}$ is a positivesemidefinite matrix, hence

$$
\left(\beta, V^{-1} A_{\mathrm{mix}}^{ \pm} V^{-1} \beta\right) \geqslant 0 \quad \text { for all } \beta \in \mathbb{R}^{2 m} .
$$

Therefore, the necessary and sufficient condition for the existence of negative values of the Wigner function is

$$
\operatorname{tr}\left(V^{-1} A_{\text {mix }}^{ \pm}\right) \geqslant 2 \text {. }
$$

By setting $\beta=0$ in (51) we clearly see that (53) is indeed a sufficient condition. Through (43), we can rephrase this condition as

$$
\begin{aligned}
& \sum_{k} \gamma_{k}\left[\left(g_{k}, V^{-1} g_{k}\right)+\left(J g_{k}, V^{-1} J g_{k}\right)\right]>2 \quad \text { (for subtraction), } \\
& \sum_{k} \gamma_{k}\left[\left(g_{k}, V^{-1} g_{k}\right)+\left(J g_{k}, V^{-1} J g_{k}\right)\right]>-2 \quad \text { (for addition), }
\end{aligned}
$$

which is automatically fulfilled for photon addition. Hence, we formally show that photon addition to a nondisplaced Gaussian state always induces a negative Wigner function, even when the initial state and the addition process are mixed. On the other hand, for photon subtraction the condition for negativity of the Wigner function can be violated when there is too much thermal noise compared to the amount of squeezing (see the example in Sec. IV A).

Finally, we emphasize that the equation

$$
\left(\beta, V^{-1} A_{\text {mix }}^{ \pm} V^{-1} \beta\right)=\operatorname{tr}\left(V^{-1} A_{\text {mix }}^{ \pm}\right)-2
$$

defines the manifold of zeros of the Wigner function. Specifically, Eq. (55) generates a multidimensional ellipsoid. The details of the manifold depend strongly on the details of the subtraction or addition process and on the covariance matrix $V$. However, as expected, the general condition for Eq. (55) to have solutions is also given by (53).

\section{Entanglement}

In this section we elaborate on the passive separability of the Wigner function (50). First, we prove that, whenever a photon is added or subtracted to or from a mode that is not entangled with any other modes in the initial Gaussian state, the resulting photon-added or -subtracted state will remain passively separable. We then prove for pure states that subtraction or addition of a photon in any other mode renders the state inherently entangled.

For any possible decomposition (23) of the Gaussian state's covariance matrix $V$, we may use (24) to write the photonsubtracted state as

$$
\begin{aligned}
\rho^{-} & =\frac{a(g) \rho_{G} a^{\dagger}(g)}{\langle\hat{n}(g)\rangle_{G}} \\
& =\frac{1}{\langle\hat{n}(g)\rangle_{G}} \int d^{2 m} \xi a(g) D(\xi) \rho_{s} D(-\xi) a^{\dagger}(g) p_{c}(\xi)
\end{aligned}
$$

and the photon-added state as

$$
\begin{aligned}
\rho^{+} & =\frac{a^{\dagger}(g) \rho_{G} a(g)}{\langle\hat{n}(g)\rangle_{G}+1} \\
& =\frac{1}{\langle\hat{n}(g)\rangle_{G}+1} \int d^{2 m} \xi a^{\dagger}(g) D(\xi) \rho_{s} D(-\xi) a(g) p_{c}(\xi),
\end{aligned}
$$


where we initially focus on the pure subtraction of a photon from mode $g \in \mathcal{N}\left(\mathbb{R}^{2 m}\right)$. The evaluation of $a(g) D(\xi) \rho_{s} D(-\xi) a^{\dagger}(g)$ is cumbersome and is therefore left for Appendix $\mathrm{C}$, where we describe the general subtraction (addition) of a photon from (to) a displaced state. In general, we can use $(\mathrm{C} 17)$ to write the Wigner function of $\rho^{ \pm}$in (56) and (57) as

$$
\begin{aligned}
W^{-}(\beta)= & \int d^{2 m} \xi W_{\xi}^{-}(\beta) \\
& \times \frac{\langle\hat{n}(g)\rangle_{s}+\frac{1}{4}\left[(\xi, g)^{2}+(\xi, J g)^{2}\right]}{\langle\hat{n}(g)\rangle_{G}} p_{c}(\xi)
\end{aligned}
$$

and

$$
\begin{aligned}
W^{+}(\beta)= & \int d^{2 m} \xi W_{\xi}^{+}(\beta) \\
& \times \frac{\langle\hat{n}(g)\rangle_{s}+1+\frac{1}{4}\left[(\xi, g)^{2}+(\xi, J g)^{2}\right]}{\langle\hat{n}(g)\rangle_{G}+1} p_{c}(\xi),
\end{aligned}
$$

with

$$
\begin{aligned}
W_{\xi}^{ \pm}(\beta)= & \frac{W_{s}(\beta-\xi)}{\operatorname{tr}\left[\left(V_{s}+\|\xi\|^{2} P_{\xi} \pm \mathbb{1}\right)\left(P_{g}+P_{J g}\right)\right]} \\
& \times\left\{\left\|\left(P_{g}+P_{J g}\right)\left(\mathbb{1} \pm V_{s}^{-1}\right)(\beta-\xi)\right\|^{2}\right. \\
& +2\left(\xi,\left(P_{g}+P_{J g}\right)\left(\mathbb{1} \pm V_{s}^{-1}\right)(\beta-\xi)\right) \\
& \left.+\operatorname{tr}\left[\left(P_{g}+P_{J g}\right)\left(\|\xi\|^{2} P_{\xi}-V_{s}^{-1} \mp \mathbb{1}\right)\right]\right\} .
\end{aligned}
$$

Note that $W_{s}$ and $V_{s}$ denote the Wigner function and covariance matrix, respectively, of $\rho_{s}$, as introduced in (24) and (25). The passive separability of the Wigner functions (58) and (59) now depends on two aspects. First, it hinges on the factorizability of the pure-state Wigner function $W_{\xi}^{ \pm}(\beta)$, as given by (60). Second, we require that

$$
p_{c}^{-}(\xi) \equiv \frac{\langle\hat{n}(g)\rangle_{s}+\frac{1}{4}\left[(\xi, g)^{2}+(\xi, J g)^{2}\right]}{\langle\hat{n}(g)\rangle_{G}} p_{c}(\xi)
$$

and

$$
p_{c}^{+}(\xi) \equiv \frac{\langle\hat{n}(g)\rangle_{s}+1+\frac{1}{4}\left[(\xi, g)^{2}+(\xi, J g)^{2}\right]}{\langle\hat{n}(g)\rangle_{G}+1} p_{c}(\xi)
$$

are well-defined probability distributions.

a. Probability distributions $p_{c}^{ \pm}(\xi)$. It is straightforwardly verified that $p_{c}^{ \pm}(\xi)$ are well-defined probability distributions. Because we know that $p_{c}^{-}$and $p_{c}^{+}$are positive functions, it suffices to validate their normalization. To do so, we evaluate

$$
\begin{aligned}
& \int d^{2 m} \xi\left[(\xi, g)^{2}+(\xi, J g)^{2}\right] p_{c}(\xi) \\
& =\int d^{2 m} \xi\left[(\xi, g)^{2}+(\xi, J g)^{2}\right] \frac{\exp \left\{-\frac{\left(\xi, V_{c}^{-1} \xi\right)}{2}\right\}}{(2 \pi)^{m} \sqrt{\operatorname{det} V_{c}}} \\
& =\left(g, V_{c} g\right)+\left(J g, V_{c} J g\right)=\operatorname{tr}\left\{\left(P_{g}+P_{J g}\right) V_{c}\right\},
\end{aligned}
$$

where we used that

$$
\int d^{2 m} \xi P_{\xi}\|\xi\|^{2} \frac{\exp \left\{-\frac{\left(\xi, V_{c}^{-1} \xi\right)}{2}\right\}}{(2 \pi)^{m} \sqrt{\operatorname{det} V_{c}}}=V_{c} .
$$

This implies that

$$
\begin{aligned}
\int d^{2 m} \xi p_{c}^{-}(\xi)= & \frac{\langle\hat{n}(g)\rangle_{s}+\frac{1}{4} \operatorname{tr}\left\{\left(P_{g}+P_{J g}\right) V_{c}\right\}}{\langle\hat{n}(g)\rangle_{G}} \\
= & \frac{1}{4\langle\hat{n}(g)\rangle_{G}}\left[\operatorname{tr}\left\{\left(P_{g}+P_{J g}\right)\left(V_{s}-\mathbb{1}\right)\right\}\right. \\
& \left.+\operatorname{tr}\left\{\left(P_{g}+P_{J g}\right) V_{c}\right\}\right] \\
= & \frac{1}{4} \frac{\operatorname{tr}\left\{\left(P_{g}+P_{J g}\right)(V-\mathbb{1})\right\}}{\langle\hat{n}(g)\rangle_{G}}=1,
\end{aligned}
$$

where we use that, by construction, $V=V_{s}+V_{c}$. Analogously, we find that

$$
\int d^{2 m} \xi p_{c}^{+}(\xi)=1
$$

b. Factorizability of $W_{\xi}^{ \pm}(\beta)$. Because $p_{c}^{ \pm}(\xi)$ are probability distributions, the states (58) and (59) are passively separable whenever a mode basis exists in which $W_{\xi}^{ \pm}(\beta)$ factorizes for every $\xi$. The factor $W_{s}(\beta-\xi)$ in (60) directly fixes a basis in which this problem must be considered because $W_{s}(\beta-\xi)$ only factorizes in a product of single-mode Wigner functions in the symplectic basis of eigenvectors of $V_{s}$. We define this basis as $\mathcal{E}_{s}=\left\{e^{(1)}, \ldots e^{(m)}, J e^{(1)}, \ldots J e^{(m)}\right\}$ and decompose the vectors $\beta$ and $\xi$ as

$$
\begin{aligned}
& \beta=\sum_{j=1}^{m} \beta_{x}^{(j)} e^{(j)}+\beta_{p}^{(j)} J e^{(j)}, \\
& \xi=\sum_{j=1}^{m} \xi_{x}^{(j)} e^{(j)}+\xi_{p}^{(j)} J e^{(j)},
\end{aligned}
$$

from which it directly follows that, in this basis, the pure-state Wigner function $W_{s}(\beta-\xi)$ takes the form

$$
W_{s}(\beta-\xi)=\prod_{j=1}^{m} W_{s}^{(j)}\left(\beta_{x}^{(j)}-\xi_{x}^{(j)}, \beta_{p}^{(j)}-\xi_{p}^{(j)}\right) .
$$

Next we consider the behavior of the polynomial

$$
\begin{aligned}
P_{2}(\beta-\xi)= & \left\{\left\|\left(P_{g}+P_{J g}\right)\left(\mathbb{1} \pm V^{-1}\right)(\beta-\xi)\right\|^{2}\right. \\
& +2\left(\xi,\left(P_{g}+P_{J g}\right)\left(\mathbb{1} \pm V^{-1}\right)(\beta-\xi)\right) \\
& \left.+\operatorname{tr}\left[\left(P_{g}+P_{J g}\right)\left(\|\xi\|^{2} P_{\xi}-V^{-1} \mp \mathbb{1}\right)\right]\right\}
\end{aligned}
$$

in this basis. The factorizability of $P_{2}(\beta-\xi)$ is completely governed by the vector $\left(P_{g}+P_{J g}\right)\left(\mathbb{1} \pm V_{s}^{-1}\right)(\beta-\xi)$. Because of the projector $\left(P_{g}+P_{J g}\right), P_{2}(\beta-\xi)$ is in essence a single-mode function determined by the mode $g$. It is straightforwardly verified that in the case in which there exists a mode $i$ in the symplectic basis of eigenvectors of $V_{s}$ for which $g \in \operatorname{span}\left\{e^{(i)}, J e^{(i)}\right\}$, we find

$$
\begin{aligned}
W_{\xi}^{ \pm}(\beta)= & W_{ \pm}^{(i)}\left(\beta_{x}^{(i)}-\xi_{x}^{(i)}, \beta_{p}^{(i)}-\xi_{p}^{(i)}\right) \\
& \times \prod_{\substack{j=1 \\
j \neq i}}^{m} W_{s}^{(j)}\left(\beta_{x}^{(j)}-\xi_{x}^{(j)}, \beta_{p}^{(j)}-\xi_{p}^{(j)}\right)
\end{aligned}
$$

such that $W_{\xi}^{ \pm}$factorizes in the mode basis $\mathcal{E}_{s}$ for any displacement $\xi$. 
We showed that the Wigner function of the single-photonadded and -subtracted states can always be represented as

$$
W^{ \pm}(\beta)=\int d^{2 m} \xi W_{\xi}^{ \pm}(\beta) p_{c}^{ \pm}(\xi)
$$

Whenever a photon is added to or subtracted from a mode that is part of a basis in which $\rho_{s}$ factorizes there exists a basis in which $W_{\xi}^{ \pm}$factorizes (71) for any $\xi$. Thus, in this case, there exists a mode basis in which the Wigner function is of the form (22), because $p_{c}^{ \pm}(\xi)$ is a well-defined probability distribution. In other words, subtracting (adding) a photon from (to) a mode that is part of a basis in which $\rho_{s}$ factorizes leads to the intuitive result that the state remains passively separable.

It follows automatically from (30) and (31) that we can generalize this approach to the scenario where the subtraction or addition process is not pure. In this case we define

$$
\lambda_{k}=\frac{\gamma_{k} \operatorname{tr}\left\{(V \pm \mathbb{1})\left(P_{g_{k}}+P_{J_{k}}\right)\right\}}{\operatorname{tr}\left\{(V \pm \mathbb{1}) \sum_{k} \gamma_{k}\left(P_{g_{k}}+P_{J g_{k}}\right)\right\}}
$$

and find that

$$
\begin{aligned}
W_{\text {mix }}^{ \pm}(\beta) & =\sum_{k} \lambda_{k} W_{g_{k}}^{ \pm}(\beta) \\
& =\int d^{2 m} \xi p_{c}^{ \pm}(\xi) \sum_{k} \lambda_{k} W_{g_{k}, \xi}^{ \pm}(\beta),
\end{aligned}
$$

where $W_{g_{k}}^{ \pm}(\beta)$ is the Wigner function of (58) or (59) for a specific subtraction mode $g_{k}$ and analogously $W_{g_{k}, \xi}^{ \pm}(\beta)$ is given by (60) for a specific subtraction (addition) mode $g_{k}$. Thus, if one can find a set of subtraction (addition) modes $\left\{g_{k}\right\}$ such that for any of these modes $g_{k} \in \operatorname{span}\left\{e^{(k)}, J e^{(k)}\right\}$, with $e^{(k)}, J e^{(k)} \in \mathcal{E}_{s}$, the state is passively separable.

Every $V_{s} \leqslant V$ gives rise to a possible decomposition (23) of the Gaussian state's covariance matrix $V$. Hence, each of these possible $V_{s}$ leads to a different basis $\mathcal{E}_{s}$ of symplectic eigenvectors with an associated set of eigenmodes. Subtracting or adding the photon in any mode in any such basis will leave the final state passively separable. In other words, the state is passively separable whenever the photon is subtracted or added in a mode that is part of any mode basis for which the initial Gaussian state is separable. It is, on the other hand, unclear that subtracting or adding a photon in any other mode automatically induces inherent entanglement. The reason is that we must consider all possible decompositions of the state $\rho^{ \pm}$in convex combinations of pure states. A priori, it is possible that convex combinations exist, which are not of the form (58) and (59). Also for such decompositions linear separability must be excluded to prove inherent entanglement. This issue falls outside of the scope of our present work and is left as an open problem.

In the special case of a pure state $V=V_{s}$ is a symplectic matrix such that we can directly find the mode basis $\mathcal{E}_{s}$ where $W_{s}(\beta-\xi)$ in (60) factorizes. If there is no mode $i$ for which $g \in \operatorname{span}\left\{e^{(i)}, J e^{(i)}\right\}$, we find that $P_{2}(\beta-\xi)$ in (70) is a sum of terms associated with different modes of the basis $\mathcal{E}_{s}$. Because $W_{\xi}^{ \pm}(\beta)$ in (60) is the Wigner function of a pure state, it is impossible to write it as statistical mixture of Wigner functions. Moreover, we cannot factorize $W_{\xi}^{ \pm}(\beta)$ in the basis where $W_{s}(\beta-\xi)$ is factorized. However, in any other basis there are cross terms in $W_{s}(\beta-\xi)$ that prevent its factorizations and are associated with off-diagonal terms in $V_{s}$ that correlate different modes. These multimode factors in $W_{s}(\beta-\xi)$ can never be compensated by terms in $P_{2}(\beta-\xi)$. Therefore, the state can never be separable in a mode basis where $W_{s}(\beta-\xi)$ does not factorize. This implies that for photon-added and -subtracted pure states the state is passively separable if and only if the subtraction or addition takes place in a mode from the mode basis for which the initial Gaussian state is separable. This mode basis coincides with the modes obtained from the Bloch-Messiah decomposition, commonly referred to as supermodes.

We stress that this implies that subtracting a photon from (or adding it to) a pure Gaussian state in a superposition of supermodes will always induce entanglement. Moreover, this entanglement is robust against linear operations in the sense that it cannot be undone by passive linear optics. Therefore, this type of inherent entanglement is clearly different from the Gaussian entanglement discussed in Sec. II E.

\section{Algebraic interpretation}

The creation of inherent entanglement due to single-photon addition and subtraction in the pure-state case can also be understood in an algebraic way. To do so, we define the operator $\mathcal{O}$ on the Hilbert space that describes the system's states, which implements a change in mode basis. On the mode space, this basis change can be implemented by the orthogonal symplectic matrix $O$. In other words, $\mathcal{O}$ describes a linear optics circuit. The action of $\mathcal{O}$ on the quadrature operators is given by

$$
\mathcal{O}^{\dagger} Q(f) \mathcal{O}=Q(O f)
$$

such that the structure of the canonical commutation relations (6) remains conserved. Because we demand $O$ to be a symplectic matrix, it follows that $O J=J O$. The definitions (28) of the creation and annihilation operators then imply that

$$
\mathcal{O}^{\dagger} a(g) \mathcal{O}=a(O g), \quad \mathcal{O}^{\dagger} a^{\dagger}(f) \mathcal{O}=a^{\dagger}(O g) .
$$

This implies also that we can write any photon subtraction given by $a(g)$ as a photon subtraction in a different mode with additional linear optic operations, since $a(g)=\mathcal{O}^{\dagger} a\left(O^{t} g\right) \mathcal{O}$. The operation $\mathcal{O}$ acts in a very natural way on a Gaussian state $\rho_{G}$, with covariance matrix $V$. Indeed, we find that $\mathcal{O} \rho_{G} \mathcal{O}^{\dagger}=$ $\rho_{G}^{\prime}$, which is a Gaussian state with covariance matrix $O V O^{t}$.

When we now consider the action of a linear optics operations $\mathcal{O}$ on the state obtained through subtracting a photon, we find

$$
\begin{aligned}
\mathcal{O} \rho^{-} \mathcal{O}^{\dagger} & =\frac{1}{\langle\hat{n}(g)\rangle_{G}} \mathcal{O} a(g) \rho_{G} a^{\dagger}(g) \mathcal{O}^{\dagger} \\
& =\frac{1}{\langle\hat{n}(g)\rangle_{G}} a\left(O^{t} g\right) \mathcal{O} \rho_{G} \mathcal{O}^{\dagger} a^{\dagger}\left(O^{t} g\right) \\
& =\frac{1}{\langle\hat{n}(g)\rangle_{G}} a\left(O^{t} g\right) \rho_{G}^{\prime} a^{\dagger}\left(O^{t} g\right) .
\end{aligned}
$$

In practice, these equalities describe very different ways of preparing an identical state as shown in Fig. 1. The discussion for photon addition is identical.

The action of linear optics (78)-(80) in the case where $O^{t} g=e^{(j)}$ such that the transformation localizes the photon 

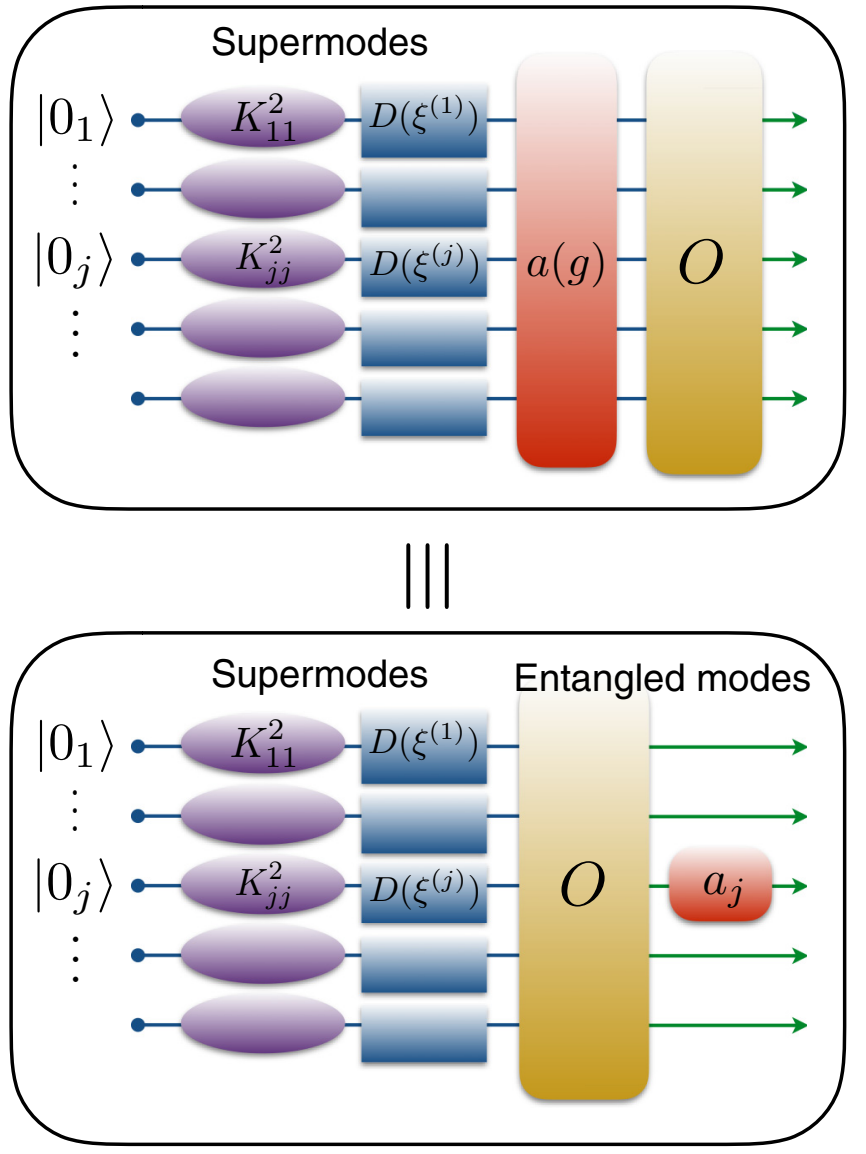

FIG. 1. Circuit representation of Eqs. (78)-(80) for a pure initial state $\rho_{G}$, represented in the mode basis obtained by the Bloch-Messiah decomposition (blue lines) as a vacuum $\left(\left|0_{1}\right\rangle \otimes \cdots \otimes\left|0_{m}\right\rangle\right)$ that is locally squeezed $K_{j j}^{2}$ and displaced $D\left(\xi^{(j)}\right)$. The top panel shows that general photon subtraction is implemented by the action of annihilation $a(g)$ in mode $g \in \mathcal{N}\left(\mathbb{R}^{2 m}\right)$, which is nonlocal (see the text) in the supermode basis. The mode basis is changed by the action of a linear optics operation (78), characterized by an orthogonal symplectic matrix $O$. The bottom panel shows the equivalent procedure (80), where the mode basis is changed (green lines) before subtracting the photon. Here we assume that $O^{t} g=e^{\prime(j)}$, which is one of the modes in the new mode basis (green lines) such that the subtraction is local. We define $a_{j}=a\left(e^{\prime(j)}\right)$.

subtraction to an entangled mode can be represented in a more graphic way, using a type of circuit representation. Specifically, in Fig. 1 we show how all the different operations act with respect to the modes where the squeezing is local (as obtained by the Bloch-Messiah decomposition). It highlights clearly that we can always find a mode basis where squeezing, displacements, and the photon subtraction (or addition) act locally. Even though experimentally one can consider copropagating modes [13], it is always possible to spatially separate these different degrees of freedom. Hence the term "local" can be physically understood in this sense.

\section{Reduced states}

An important tool to measure entanglement in quantum systems is the reduced quantum state. When we study entanglement, we typically fix a partition of the system to entangle (in the case of multimode quantum optics, this partition is comprised of different modes). The reduced state is obtained by integrating (or tracing) out several of these degrees of freedom. These reduced states are important in the study of entanglement properties, specifically when the full state is pure.

The methods provided in Sec. III B are ideally suited to derive the Wigner functions for the reduced states of a multimode photon-added or -subtracted state. In particular, we stress that the characteristic function (9) is in principle obtained in a single-mode fashion. Therefore, we can obtain the characteristic function of the reduced state, associated with a mode space $\mathcal{M} \subset \mathcal{N}\left(\mathbb{R}^{2 m}\right)$, by simply restricting $\alpha$ in (9) to $\alpha=\lambda f$ with $f \in \mathcal{M}$ and $\lambda \in \mathbb{R}$.

We can now define a symplectic basis $\mathcal{E}_{\mathcal{M}}=$ $\left\{v^{(1)}, \ldots, v^{\left(m^{\prime}\right)}, J v^{(1)}, \ldots, J v^{\left(m^{\prime}\right)}\right\}$ of $\mathcal{M}$, where $\operatorname{dim} \mathcal{M}=$ $m^{\prime}<m$. The restrictions of the matrices $V$ and $A_{\text {mix }}^{ \pm}$to $\mathcal{M}$ are denoted by $V^{\mathcal{M}}$ and $A_{\text {mix }}^{\mathcal{M}}$, respectively. These restricted matrices are the ones that are obtained by only measuring the correlations among the modes in $\mathcal{M}$, which directly follows from (42) and (44). This straightforwardly implies that the Wigner function of the state on the reduced mode set $\mathcal{M}$ has the form

$$
\begin{aligned}
W_{\mathcal{M}}^{ \pm}\left(\beta^{\prime}\right)= & \frac{1}{2}\left[\left(\beta^{\prime}, V^{\mathcal{M}^{-1}} A_{\text {mix }}^{\mathcal{M}} V^{\mathcal{M}^{-1}} \beta^{\prime}\right)\right. \\
& \left.-\operatorname{tr}\left(V^{\mathcal{M}^{-1}} A_{\text {mix }}^{\mathcal{M}}\right)+2\right] \\
& \times \frac{1}{(2 \pi)^{m} \sqrt{\operatorname{det} V^{\mathcal{M}}}} e^{-\left(\beta^{\prime}, V^{\mathcal{M}^{-1}} \beta^{\prime}\right) / 2},
\end{aligned}
$$

where $\beta^{\prime}$ is a vector in the optical phase space $\beta^{\prime} \in \mathbb{R}^{2 m^{\prime}}$ associated with $\mathcal{M}$.

The Wigner function of the reduced state naturally provides us with a measure for the entanglement of the state if the initial $m$-mode state is pure. Indeed, selecting a set of modes determines a bipartition that contains the modes $\mathcal{M}$ on the one hand and the modes that were integrated out, $\mathcal{M}^{\perp}$, on the other hand. The purity of the reduced state on the modes $\mathcal{M}$ is directly related to the entanglement between the modes in $\mathcal{M}$ and in $\mathcal{M}^{\perp}$. The less pure the reduced state, the higher the entanglement between the two parts of the bipartition.

Once the Wigner function of the reduced state is obtained, its purity can directly be evaluated. It is given by

$$
\mu_{\mathcal{M}}=(4 \pi)^{m^{\prime}} \int_{\mathbb{R}^{2 m^{\prime}}} d^{2 m^{\prime}} \beta^{\prime}\left[W_{\mathcal{M}}^{ \pm}\left(\beta^{\prime}\right)\right]^{2} .
$$

In the following section, we will use (82) to evaluate the purestate results obtained in Sec. III B 3.

\section{EXAMPLES}

In the preceding sections we developed a framework for the analysis of single-photon added and subtracted states. The goal of this section is to provide two examples to highlight the usefulness of the above results. In the first example, we treat the well-known case of a two-mode squeezed vacuum, where both modes are equally squeezed. The second example extends the study in [29] and uses an initial Gaussian state that was experimentally obtained [36]. 

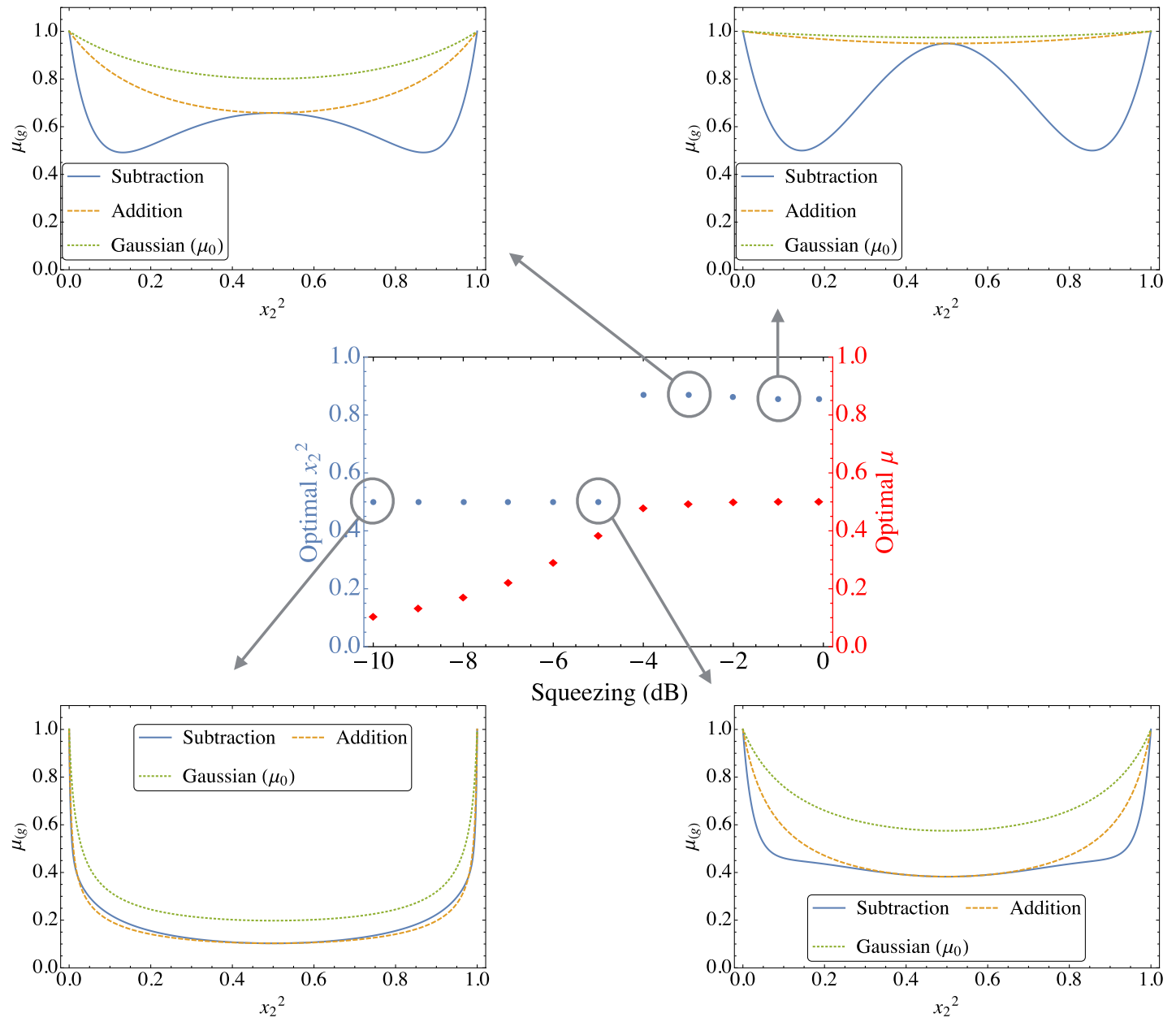

FIG. 2. Exterior panels show the purity (82) of the state reduced to the mode $g=\left(x_{1}, 0,0, x_{2}\right)^{t}$, in which the photon was added (orange dashed curve) or subtracted (blue solid curve), as a function of the weight $x_{2}^{2}$ in the superposition of supermodes. The degree of squeezing, i.e., $s$ in (83), is set to $s=1 \mathrm{~dB}$ (top right), $s=3 \mathrm{~dB}$ (top left), $s=5 \mathrm{~dB}$ (bottom right), and $s=10 \mathrm{~dB}$ (bottom left). The purity of the reduced Gaussian state, in the same mode, is shown as a reference (green dotted curve). The lower purity is directly related to higher entanglement between $g$ and the complementary orthogonal mode. The center panel shows values of the weight $x_{2}^{2}$ (blue dots, left vertical axis), with $g=\left(x_{1}, 0,0, x_{2}\right)^{t}$, for which the lowest purity (red diamonds, right vertical axis), and thus the highest entanglement, is achieved for different values of squeezing in the case of photon subtraction. Points associated with exterior panels are highlighted.

\section{A. Two-mode symmetrically squeezed vacuum}

The two-mode symmetrically squeezed vacuum state is in a certain sense the simplest nonclassical multimode Gaussian state. We characterize it through its covariance matrix $V_{s}$, which takes the form

$$
V_{s}=\left(\begin{array}{cccc}
10^{-s / 10} & 0 & 0 & 0 \\
0 & 10^{-s / 10} & 0 & 0 \\
0 & 0 & 10^{s / 10} & 0 \\
0 & 0 & 0 & 10^{s / 10}
\end{array}\right)
$$

in its basis of eigenmodes. The notation is chosen such that $s$ denotes the amount of squeezing in decibels.

At first, we use the results presented in Secs. III B and III D to investigate the entanglement properties that are induced by adding or subtracting a photon. With the covariance matrix (83) we have all the necessary information to construct the Wigner function (50) that describes a state with a single photon added or subtracted in mode $g \in \mathcal{N}\left(\mathbb{R}^{4}\right)$. To induce entanglement, we must subtract a photon in a superposition of two supermodes. Due to the symmetry properties of the state, it is also important to include a phase in this superposition. Hence, the most interesting choice is $g=\left(x_{1}, 0,0, x_{2}\right)^{t}$ with $x_{1}^{2}+x_{2}^{2}=1$.

In Fig. 2 we investigate the purity $\mu$ [Eq. (82)] for the reduce density matrix associated with the mode where the photon is subtracted or added. This is in essence a matter of integrating out the mode orthogonal to $g$ or setting $\mathcal{M}=\operatorname{span}\{g, J g\}$ in (82). In practice, we analytically construct the reduced state's Wigner function (81) and perform a numerical integration to obtain the purity, denoted by $\mu_{(g)}$. Because the purity of the reduce state is directly related to an entanglement measure if the initial state is pure, we can directly associate $\mu_{(g)}$ with the entanglement between $g$ and the complementary mode. Figure 2 investigates what happens to the entanglement as we vary the only parameter left in the system, $x_{2} / x_{1}$, which governs $g$, and the squeezing $s$ in (83). 
As a reference, let us first consider entanglement in the Gaussian state before the subtraction or addition has taken place. We observe entanglement between the mode $\mathcal{M}=$ $\operatorname{span}\{g, J g\}$ and the complementary mode $\mathcal{M}^{\perp}$ in the initial squeezed vacuum state, characterized by (83). It can be clearly seen in Fig. 2 (green curves) that in the Gaussian state entanglement is maximal in the balanced case, i.e., for $x_{2}^{2}=$ $1 / 2$. Moreover, we observe that this Gaussian entanglement increases with increased squeezing. The results for photon addition (orange curves) are qualitatively the same as the Gaussian case. However, quantitatively, the obtained purities are lower, implying that the addition of a photon increases entanglement.

The results for photon subtraction in Fig. 2 (blue curves and central panel) are more surprising. For high squeezing $s>5 \mathrm{~dB}$, we observe a similarity with the results for photon addition. However, for low squeezing $s<5 \mathrm{~dB}$, the lowest purity is no longer obtained for the balanced superposition of supermodes $x_{2}^{2}=1 / 2$, but rather for the highly imbalanced superposition with $x_{2}^{2} \approx 0.85 .{ }^{5}$ Due to the symmetry in the squeezing, the system is completely unchanged when $x_{1}$ and $x_{2}$ are interchanged. This implies that exactly the same value for the purity is obtained for $x_{2}^{2} \approx 0.15$. It is particularly surprising that, even for $s=1$ in (83), we still observe that $\mu_{(g)}=0.5$ when $x_{2}^{2} \approx 0.85$. Hence, we highlight a profound difference in the induced entanglement properties for the subtraction as compared to the addition of a photon.

In the limit of low squeezing (i.e., $s \rightarrow 0$ ) it is useful to analyze the state that is obtained by photon subtraction in the photon representation. We straightforwardly obtain that, in the low squeezing limit, the state, denoted by $\left|\psi^{-}\right\rangle$, is given by

$$
\left|\psi^{-}\right\rangle=\frac{2\left(x_{1}^{2}-x_{2}^{2}\right)|1,0\rangle+4 x_{1} x_{2}|0,1\rangle}{\sqrt{4\left(x_{1}^{2}-x_{2}^{2}\right)^{2}+16 x_{1}^{2} x_{2}^{2}}},
$$

where $\left|n_{1}, n_{2}\right\rangle$ is the state with $n_{1}$ photon in mode 1 and $n_{2}$ photons in mode 2 . It is directly verified that for any solution with $x_{1}^{2}=1-x_{2}^{2}$ and $x_{2}^{2}=(2 \pm \sqrt{2}) / 4$, the state $\left|\psi^{-}\right\rangle$is a single-photon Bell state. This immediately gives the reason why we observe a reduced state purity of $1 / 2$ for these modes. The most remarkable aspect of our results is that we show how this observed entanglement between the modes survives under reasonably high amounts of squeezing, where both modes are populated with many photons. Experimentally, this should make this phenomenon easier to observe. Note that our result is in agreement with other theoretical works [44,45], where different mathematical tools were used to characterize the entanglement induced by photon subtraction from the two-mode symmetrically squeezed vacuum in the low squeezing limit.

Not only the entanglement properties, but also the negativity of the Wigner function behaves very differently for photonadded and -subtracted states. We probe the state's negativity through the witness (53) and immediately observe that, for both addition and subtraction of a photon, $\operatorname{tr}\left(V^{-1} A_{g}^{ \pm}\right)=4$

\footnotetext{
${ }^{5}$ The exact value of $x_{2}^{2}$ for which the minimal purity is achieved weakly varies with the squeezing.
}
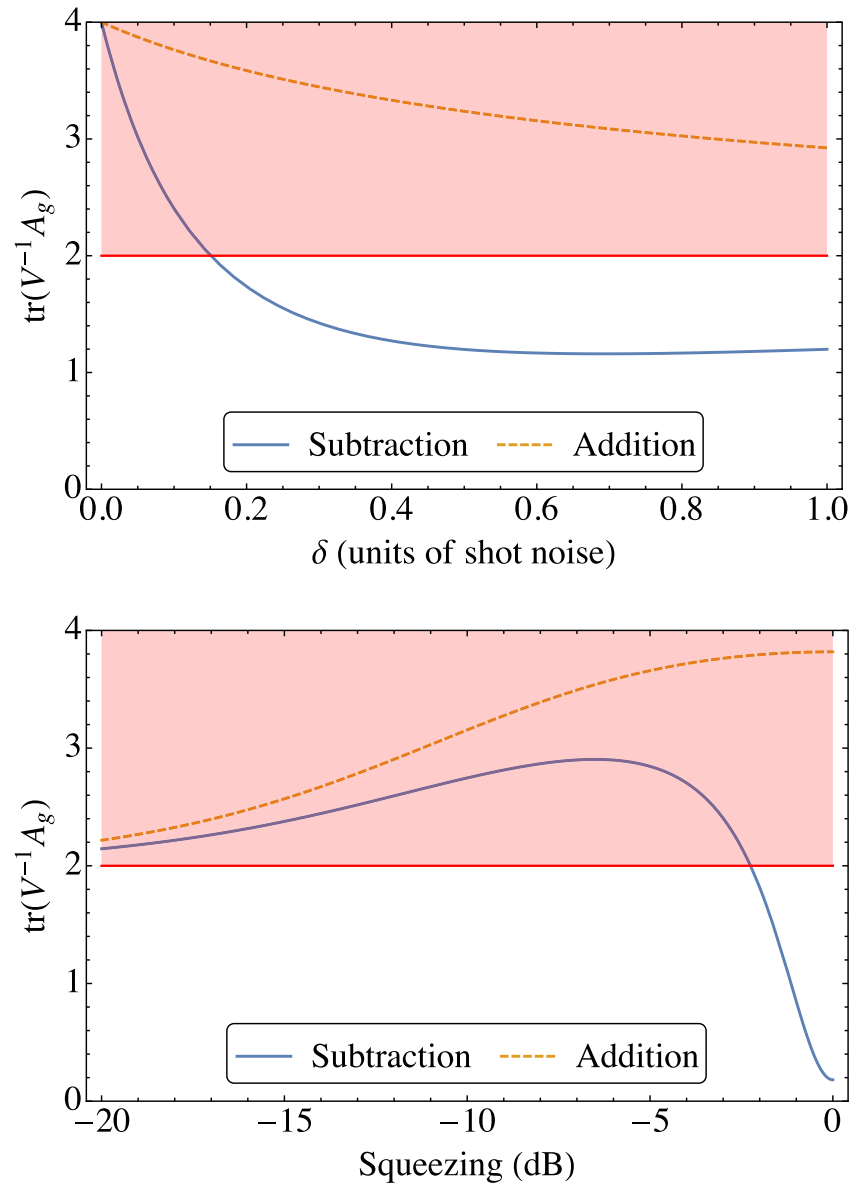

FIG. 3. Negativity condition (53) (red highlighted region) shown as a function of the classical noise $\delta(85)$ for a fixed squeezing of $3 \mathrm{~dB}$ (top) and as a function of squeezing for a fixed noised of $\delta=0.1$ as a fraction of the shot noise (bottom). The mode in which addition (orange dashed curve) and subtraction (blue solid curve) take place is set to $g=(1 / \sqrt{2}, 0,0,1 / \sqrt{2})^{t}$.

when the state is pure. Hence, the condition (53) for negativity is satisfied such that the Wigner function is negative. More interesting is the more general case of addition or subtraction from a mixed state. We approach this scenario through a simple noise model by considering an initial Gaussian state with a covariance matrix

$$
V=V_{s}+\delta \mathbb{1},
$$

where $\delta$ indicates the amount of added classical noise relative to the shot noise. In Fig. 3 we show how the negativity witness $\operatorname{tr}\left(V^{-1} A_{g}^{ \pm}\right)$is influenced by the variation of the noise $\delta$ and of the squeezing $s$ in (83). As derived in (54), we observe that (53) is always fulfilled for photon addition. In contrast, the negativity of the Wigner function for photon subtraction is very sensitive to the added noise $\delta$. It is not surprising that states that are more strongly squeezed are more robust to noise. Finally, we note that, due to the symmetry of the squeezing in both supermodes, $\operatorname{tr}\left(V^{-1} A_{g}^{ \pm}\right)$is fully independent of the mode (or mixture of modes) in which the photon is added or subtracted.

In summary, we highlighted the potential of the methods of Sec. III B to study the photon addition and subtraction from a 
symmetric two mode squeezed vacuum. We showed that pure photon-subtracted states have highly interesting entanglement properties in the regime of low squeezing. Nevertheless, the negativity of the Wigner function, which is a crucial property to reach a quantum advantage in computation, is much more sensitive to noise for photon subtraction than for photon addition. A detailed understanding of the interplay of these negativities and the entanglement properties of the states lies beyond the scope of this work.

\section{B. Experimentally generated Gaussian state}

In [29] we already presented results for an initial Gaussian state that was experimentally obtained [36]. Here we complement these results with, on the one hand, additional findings for the entanglement between modes in the pure-state part. On the other hand, we provide a study of the effect of impure addition and subtraction [in the sense of (40) and (41)] on the negativity of the resulting Wigner function.

\section{Inherent entanglement}

Our study of inherent entanglement is restricted to pure states, in accord with Sec. III B 3. However, the covariance matrix $V$ of [36] is not symplectic, i.e., we cannot find a symplectic basis of eigenvectors, which directly implies that the state cannot be pure. This can explicitly be seen in the Williamson decomposition $V=S^{t} \Delta S$, where $S$ is symplectic and $\Delta \geqslant \mathbb{1}$ is a diagonal matrix. For a pure state, one must find that $\Delta=\mathbb{1}$, which is not the case for $V$. Hence, we will investigate entanglement properties for photon addition and subtraction from a pure squeezed vacuum, which is consistent with $V$. To do so, we resort to the Bloch-Messiah decomposition $S=O^{\prime} K O$, where $O$ and $O^{\prime}$ are orthogonal symplectic matrices and $K$ is a positive diagonal symplectic matrix. We then obtain that $V=O^{t} K O^{\prime t} \Delta O^{\prime} K O$, which we use to decompose $V=V_{s}+V_{c}$ as in Sec. IIE. Through this method, we obtained a squeezed vacuum that is characterized by $V_{s}=K^{2}$ in the basis of eigenmodes.

Our previous work [29] showed that photon addition or subtraction can increase entanglement between the mode in which the photon is added or subtracted and the additional modes. The results in Sec. III B 3 show, moreover, that the subtraction or addition of a photon in a superposition of eigenmodes of $V_{s}$ induces entanglement in every possible mode basis. Here, in Figs. 4 and 5, we illustrate this point via the reduced state purity as a probe of the entanglement in specific bipartitions.

In both Figs. 4 and 5 a photon is subtracted from or added to the squeezed vacuum of a random mode $g \in \mathcal{N}\left(\mathbb{R}^{2 m}\right)$. First, we subtract or add a photon to the squeezed vacuum and reduce the obtained state to a randomly chosen mode $f$, as in Sec. IIID. The purity $\mu$ of these reduced photon-added and -subtracted states is compared to the purity $\mu_{0}$ of the reduced squeezed vacuum prior to the addition or subtraction of a photon for the same mode $f$. By probing 1000 different random choices for $f$, Fig. 4 shows entanglement for every bipartition of a randomly chosen mode $f \in \mathcal{N}\left(\mathbb{R}^{2 m}\right)$ and the 15 complementary modes. More notably, we observe that the purity $\mu$ of the reduced photon-added and -subtracted states is always lower than the Gaussian state's purity $\mu_{0}$. This is

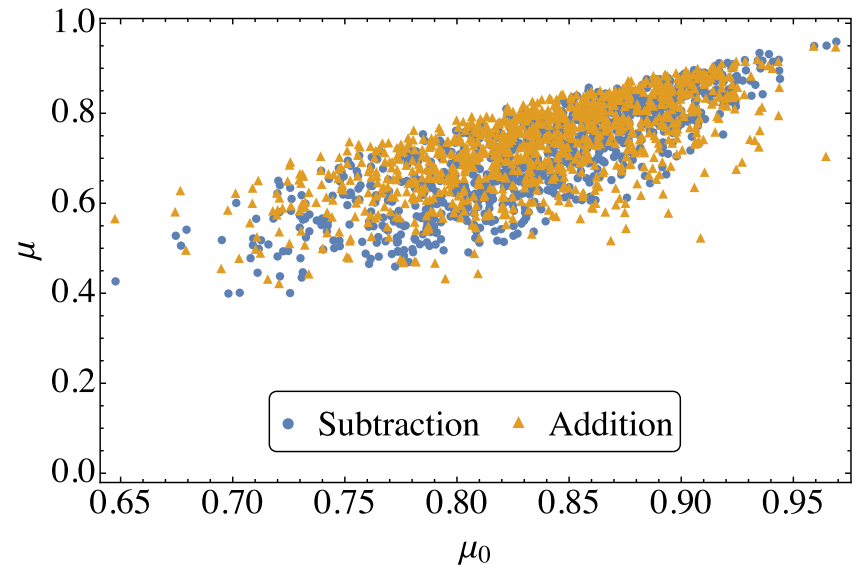

FIG. 4. Purity $\mu$ (82) of the single-mode reduced state after the addition (orange triangles) or subtraction (blue dots) of a photon from a pure state deduced (see the text) from the experimental state [36]. Purities $\mu$ are compared to the purity $\mu_{0}$ of the same mode's reduced state before the addition or subtraction, i.e., the initial Gaussian state. For all realizations, the photon is added or subtracted in the same randomly chosen mode $g \in \mathcal{N}\left(\mathbb{R}^{32}\right)$. The mode $f \in \mathcal{N}\left(\mathbb{R}^{32}\right)$ to which the state is reduced is chosen randomly for each realization. Points where $\mu=\mu_{0}$ are indicated by the red solid curve.

compelling numerical evidence that, for any squeezed vacuum and any given mode basis, entanglement never decreases through the addition or subtraction of a photon.

According to Sec. III B 3, we must observe entanglement in every mode basis, provided the mode in which the photon is subtracted or added is not an eigenmode of the squeezed vacuum, i.e., $g$ is not an eigenvector of $V_{s}$. Figure 4 indicates that photon subtraction and addition increase entanglement

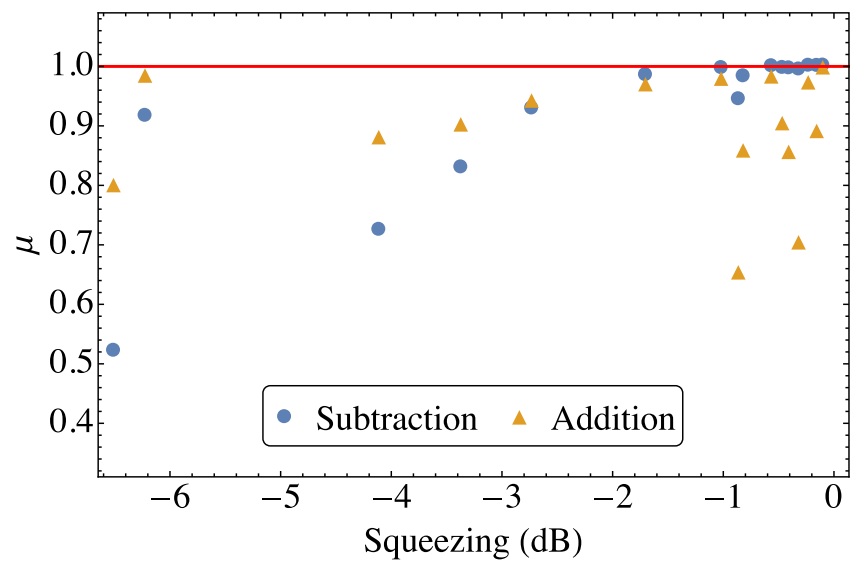

FIG. 5. Purity $\mu$ (82) of the single-mode reduced state after the addition (orange triangles) or subtraction (blue dots) of a photon in a pure state deduced (see the text) from the experimental state [36]. Each data point represents the reduction of the state to a different eigenmode of the initial Gaussian state's covariance matrix. The horizontal axis indicates the squeezing (in $\mathrm{dB}$ ) of each of these eigenmodes. For all data points, the photon is added or subtracted in the same randomly chosen mode $g \in \mathcal{N}\left(\mathbb{R}^{32}\right)$. The initial Gaussian state is fully separable from the considered mode basis, hence the red curve indicates $\mu=1$, in analogy with the red solid curve in Fig. 4. 
as compared to the initial Gaussian state. This implies that any bipartition of modes that is entangled for the initial Gaussian state will remain entangled after the addition or subtraction process. Hence, it remains to verify the presence of entanglement in the basis eigenmodes of the squeezed vacuum where the initial Gaussian state is fully separable (this basis is unique because we consider a pure state with nondegenerate squeezing). This scenario is considered in Fig. 5, where the photon-subtracted and -added squeezed vacuums are reduced to each of the different eigenmodes of $V_{s}$, sorted according to their respective squeezing. It can be seen that photon addition and subtraction entangle a bipartition of a significantly squeezed significantly squeezed mode and the complementary modes. We note, moreover, that photon subtraction generates more entanglement than photon addition whenever the squeezing is sufficiently high (which is consistent with other recent studies [46]). Modes with very low squeezing can essentially be interpreted as the vacuum, which limits the effectiveness of photon subtraction.

It must be emphasized that all modes in Fig. 5 are part of the same mode basis and that the mode in which the photon is added or subtracted is fixed. Therefore, Fig. 5 highlights that the state is not fully separable in the basis of eigenmodes of $V_{s}$, i.e., its Wigner function cannot be written as (22). The results in Fig. 4 indicate the presence of entanglement in any other mode basis. Hence, we have failed to find any basis in which the photon-added or -subtracted state is fully separable. This is consistent with the state being inherently entangled, as predicted in Sec. III B 3.

\section{Negativity}

The negativity of the Wigner function obtained through the pure addition or subtraction of a photon to the Gaussian state of [36] was already studied in [29]. Here we treat the case where the addition and subtraction processes are impure, with $A_{\text {mix }}^{ \pm}$given by (43). In Fig. 6 we probe the negativity witness $\operatorname{tr}\left(V^{-1} A_{\text {mix }}^{ \pm}\right)$for a varying degree of impurity in the addition and subtraction processes. The top panel shows the case for pure subtraction as a reference. Every data point corresponds to one randomly generated mode $g \in \mathcal{N}\left(\mathbb{R}^{32}\right)$, in which the photon is subtracted or added, for which $\operatorname{tr}\left(V^{-1} A_{\text {mix }}^{ \pm}\right)$was evaluated.

Descending through the panels of Fig. 6, the impurity of photon addition and subtraction is increased. For the second and third plot from the top, five and ten random orthogonal modes, respectively, participate in the process. In practice, this implies the choice of a set $\left\{g_{1}, \ldots, g_{10}\right\}$ of random orthogonal vectors in $\mathcal{N}\left(\mathbb{R}^{32}\right)$. Following (40) and (41), each of these modes $g_{k}$ comes with an associated weight $\gamma_{k}$, which physically quantifies the probability that a subtracted photon originated from the associated mode (or that the photon is added to the associated mode in the case of addition). For the sake of simplicity, we choose these weights to be uniform over the modes. Thus, we set $\gamma_{k}=1 / 5$ for the mixture of five modes (second panel from the top in Fig. 6) and $\gamma_{k}=1 / 10$ in the mixture of ten modes (third panel from the top in Fig. 6).

We observe that the data points are less scattered for increasing amounts of impurities. This should not come as a surprise, because increasing impurity implies an averaging
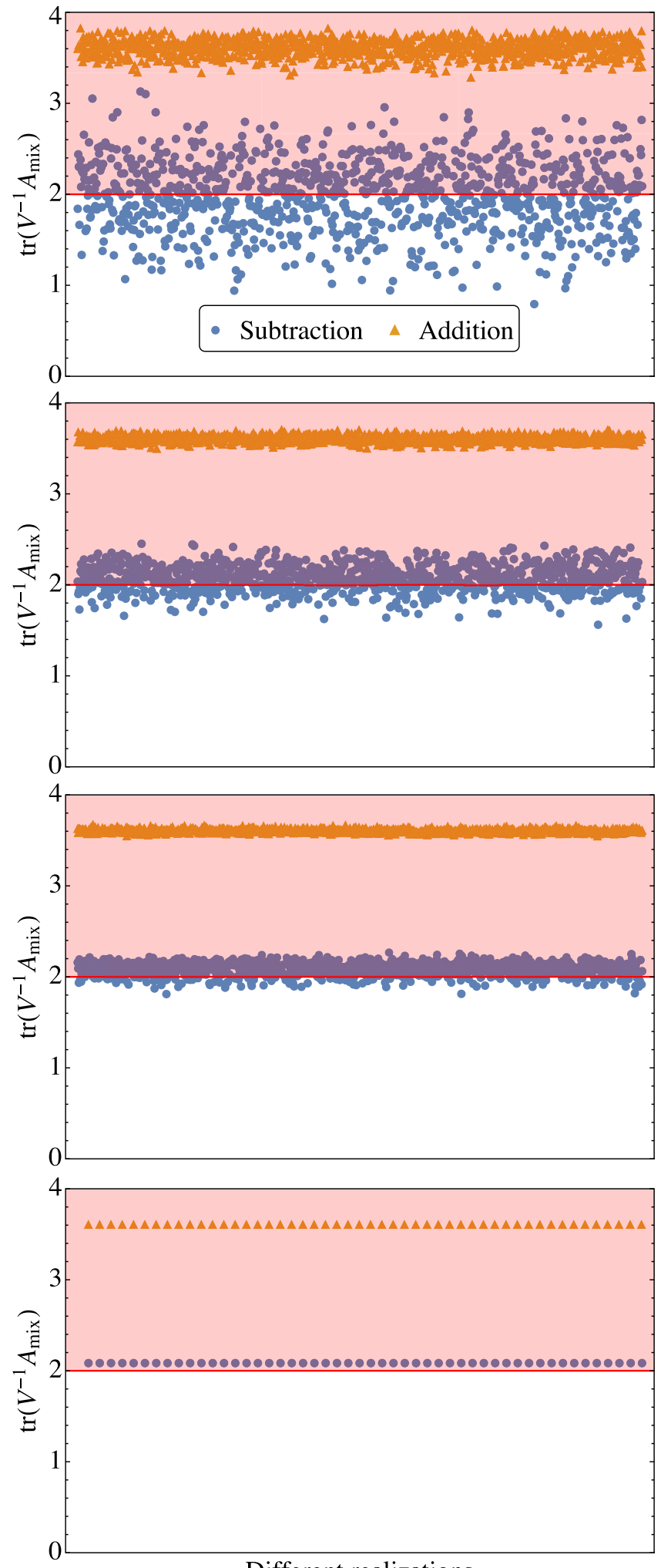

Different realizations

FIG. 6. Test of the negativity condition (53) for an experimentally obtained state [36], with photon subtraction or addition in a random mode (top) or a mixture of random orthogonal modes (other panels). Every data point represents a random choice of one (top), five (second from top), ten (second from bottom), or 16 (bottom) orthogonal modes $g_{k}$ in the mixtures (30) and (31). Weights $\gamma_{k}$ in the mixtures (30) and (31) are the same for every mode. Only realizations falling in the red highlighted zone lead to negative Wigner functions. 
over the randomly chosen modes. This leads to the expectation that for 16 random orthogonal modes, all realizations should coincide, which is confirmed in the bottom panel of Fig. 6. In this case, for every data point, a photon is subtracted from or added to a balanced mixture of all modes such that this scenario describes a fully mode-independent photon addition or subtraction. Physically, this case is particularly relevant as for photon subtraction it corresponds to the use of a beam splitter on a set of copropagating modes.

In this case we find that $A_{\mathrm{mix}}^{ \pm}$is independent of the choice of basis in which the mixture $\left\{g_{k}\right\}$ is represented. Hence, none of the properties of the photon-added or-subtracted state depends on the mode basis. From (43), we directly obtain that fully mixed subtraction or addition leads to

$$
\operatorname{tr}\left(V^{-1} A_{\mathrm{mix}}^{ \pm}\right)=2\left(1+\frac{\operatorname{tr}\left(V^{-1} \pm \mathbb{1}\right)}{\operatorname{tr}(V \pm \mathbb{1})}\right)
$$

Furthermore, in the fully mode-independent scenario, the negativity condition (53) reduces to

$$
\operatorname{tr}\left(V^{-1} \pm \mathbb{1}\right)>0
$$

In the case of $V$ obtained from [36], we obtain that $\operatorname{tr}\left(V^{-1}-\right.$ $\mathbb{1})=0.672702$ for photon subtraction, which clearly satisfies the condition (87). It is remarkable that a highly multimode impure experimentally generated state can still lead to a negative Wigner function upon photon subtraction with a simple beam-splitter setup. We stress, however, that our result refers to the negativity of the full multimode Wigner function, which is difficult to observe in single-mode measurements. Moreover, Fig. 6 shows that $\operatorname{tr}\left(V^{-1} A_{\text {mix }}^{ \pm}\right)$is only slightly larger than 2. This suggests that the region in phase space where the Wigner function becomes negative is small compared to that of a pure photon-subtracted state.

Hence, we showed that experimentally achieved Gaussian states can lead to negative Wigner functions upon photon subtraction. In the case of photon addition, negativity of the Wigner function is taken for granted, as implied by (54). Furthermore, we showed that the entanglement properties of the pure squeezed vacuum, extracted from the Gaussian state [36] through the Williamson and Bloch-Messiah decompositions, are in agreement with the results in Sec. III B 3. It remains an open question whether these entanglement properties persist and can be used when we consider the actual mixed state that is obtained in the experiment.

\section{CONCLUSION}

\section{A. Summary}

We started the analysis of nondisplaced multimode photonadded and -subtracted states (where the addition or subtraction is not necessarily pure) by deriving their truncated correlations functions (44) of arbitrary order. The truncated correlations as such suffice to characterize any quantum state. For Gaussian states, in particular, these truncated correlations vanish beyond second order. Hence, we can interpret all truncated correlations beyond second order as clear signatures of the non-Gaussian properties of the state.

However, in this work the truncated correlations were primarily used as a tool to derive the full-state Wigner function for multimode photon-added and -subtracted states (50). This result is also highlighted in [29] and provides a compact and insightful description of a nondisplaced multimode photon-added and -subtracted state with arbitrarily many modes. The more general (and more cumbersome) result for subtraction and addition from a displaced Gaussian state is given in Appendix C.

The non-Gaussian properties of the photon-added and -subtracted states are all encrypted in the polynomial part of the Wigner function (50). In particular, we obtained an elegant and simple condition (53) for having a negative Wigner function. Notably, this condition can be used as a tool for selecting the mode in which to subtract a photon. For photon addition, on the other hand, we formally proved that this condition is always fulfilled such that the Wigner function is always negative. This negativity condition was studied for the concrete examples of a two-mode symmetrically squeezed vacuum and an experimentally obtained Gaussian state in Figs. 3 and 6, respectively.

Finally, we devoted a considerable part of this work to the study of the entanglement properties that can be extracted from the Wigner function (50). In Sec. III B 3 we formalized that subtracting (or adding) a photon in a mode that is part of a mode basis in which the initial Gaussian state is separable will leave the photon-subtracted (or -added) state separable. For pure states, we showed that subtraction (or addition) of the photon in any other mode will induce entanglement. Importantly, this entanglement cannot be undone by passive linear optics operations. In contrast, we stress in Sec. II E that any entanglement in a Gaussian state can always be undone by changing the mode basis through a passive linear optics operation. Furthermore, we evaluated the reduction of the Wigner function to a subset of modes (81). For a global pure state, the purity of this reduced state can then be used as a quantitative probe for the entanglement between the mode to which the system is reduced and the modes that were integrated out. These pure-state entanglement properties were evaluated in Figs. 2 and in Figs. 4 and 5 for a two-mode symmetrically squeezed vacuum and a 16-mode squeezed vacuum that is compatible with the experimentally obtained Gaussian state of [36], respectively.

\section{B. Outlook}

We started our Introduction by emphasizing the importance of non-Gaussian states for quantum computation. In this work we have developed a set of tools that is ready to approach concrete quantum information problems and quantum optics experiments. Notably, one may use these techniques for a detailed analysis of single-photon subtraction from a CV cluster state, as used in measurement-based quantum computation [47].

The presented results also impose several open questions for future research. First, there are still properties of the general Wigner function (50) that are to be unveiled. Most notably, we think about the negativity volume of the Wigner function, i.e., the integral of the negative part. We conjecture that the quantity $\operatorname{tr}\left(V^{-1} A_{\text {mix }}^{ \pm}\right)$in (53) will be proportional to the negativity volume, but this remains to be proven. Moreover, it remains to be understood how a photon subtraction or addition in a particular mode locally affects different modes as represented by the reduction of the Wigner function to these modes (81).

A second open question is the generalization of our entanglement results to mixed states. As discussed in Sec. III B 3, 
this is not expected to be a straightforward task. A potential route may be to derive bounds on the entanglement in the spirit of [48].

Finally, it remains an open question how our present results generalize to multiphoton addition and subtraction. A priori, the methods applied here still apply in a more general scenario, but the derivation of the truncated correlations is expected to become a formidable task. Nevertheless, these truncated correlations are a crucial element of our study, because they are directly measurable in state-of-the-art experiments [36].

\section{ACKNOWLEDGMENT}

This work was supported by the French National Research Agency projects COMB and SPOCQ and the European Union Grant QCUMbER (No. 665148). C.F. and N.T. acknowledge financial support from the Institut Universitaire de France.

\section{APPENDIX A: DERIVATION TRUNCATED CORRELATION FUNCTIONS}

We prove Eq. (44) by induction on $k$, which implies that we assume that all even-order truncated correlations up to order $2 k-2$ are indeed given by (44). Moreover, we use that for $k=1$ we have the addition expression (38), which was derived explicitly, and that all odd-order truncated correlations vanish.

We start by explicitly writing that

$$
\left\langle Q\left(f_{1}\right) \cdots Q\left(f_{2 k}\right)\right\rangle_{T}=\operatorname{tr}\left\{\rho Q\left(f_{1}\right) \cdots Q\left(f_{2 k}\right)\right\}-\sum_{p \in \mathcal{P}^{(2,4, \ldots, 2 k-2)}} \prod_{i \in p}\left\langle Q\left(f_{i_{1}}\right) \cdots Q\left(f_{i_{r}}\right)\right\rangle_{T},
$$

where we use the notation $\mathcal{P}^{(2,4, \ldots, 2 k-2)}$ to indicate the set of all partitions of the index set $\{1, \ldots, 2 k\}$ where the allowed number of elements in the subsets (that constitute the partitions) is $2,4, \ldots, 2 k-2$ (all even orders up to $2 k-2$ ). This implies that we for a partition $p \in \mathcal{P}^{(2,4, \ldots, 2 k-2)}$, we cannot fix the number over element in a subset $i \in p$. Therefore, we denote this number of elements by $r$, where we know that $r$ is even and smaller than or equal to $2 k-2$. Therefore, we know that

$$
\left\langle Q\left(f_{i_{1}}\right) \cdots Q\left(f_{i_{r}}\right)\right\rangle_{T}=\delta_{2, r}\left\langle Q\left(f_{i_{1}}\right) Q\left(f_{i_{2}}\right)\right\rangle_{G}+(-1)^{r / 2-1}(r / 2-1) ! \sum_{p^{\prime} \in \mathcal{P}_{i}^{(2)}} \prod_{i^{\prime} \in p^{\prime}}\left(f_{i_{1}^{\prime}}, A_{\mathrm{mix}}^{ \pm} f_{i_{2}^{\prime}}\right),
$$

with $\delta_{2, r}$ the Kronecker delta. Moreover, we are now considering $\mathcal{P}_{i}^{(2)}$ as the set of partitions of the index set $\left\{i_{1}, \ldots, i_{r}\right\}$.

Furthermore, using the factorization properties of a Gaussian state, we can express the term

$$
\begin{aligned}
\operatorname{tr}\left\{\rho Q\left(f_{1}\right) \ldots Q\left(f_{2 k}\right)\right\} & =\left\langle Q\left(f_{1}\right) \ldots Q\left(f_{2 k}\right)\right\rangle_{G}+\sum_{p \in \mathcal{P}^{(2)}} \sum_{i \in p}\left(\left(f_{i_{1}}, A_{\text {mix }}^{ \pm} f_{i_{2}}\right) \prod_{j \in p \backslash i}\left\langle Q\left(f_{j_{1}}\right) Q\left(f_{j_{2}}\right)\right\rangle_{G}\right) \\
& =\sum_{p \in \mathcal{P}^{(2)}} \prod_{i \in p}\left\langle Q\left(f_{i_{1}}\right) Q\left(f_{i_{2}}\right)\right\rangle_{G}+\sum_{p \in \mathcal{P}^{(2)}} \sum_{i \in p}\left(\left(f_{i_{1}}, A_{\text {mix }}^{ \pm} f_{i_{2}}\right) \prod_{j \in p \backslash i}\left\langle Q\left(f_{j_{1}}\right) Q\left(f_{j_{2}}\right)\right\rangle_{G}\right),
\end{aligned}
$$

from which it is clear that $\left\langle Q\left(f_{1}\right) \cdots Q\left(f_{2 k}\right)\right\rangle_{T}$ contains products of up to $k$ copies of $\left(f_{i_{1}}, A_{\text {mix }}^{ \pm} f_{i_{2}}\right)$ (where the arguments $f_{i_{1}}$ and $f_{i_{2}}$ vary).

To determine the expression for this product, we first focus on the terms that have exactly $k$ copies of $\left(f_{i_{1}}, A_{\text {mix }}^{ \pm} f_{i_{2}}\right)$. These terms are all contained within

$$
-\sum_{p \in \mathcal{P}^{(2,4, \ldots, 2 k-2)}} \prod_{i \in p}\left\langle Q\left(f_{i_{1}}\right) \cdots Q\left(f_{i_{r}}\right)\right\rangle_{T}=-\sum_{p \in \mathcal{P}^{(2,4, \ldots, 2 k-2)}} \prod_{i \in p}\left(\delta_{2, r}\left\langle Q\left(f_{i_{1}}\right) Q\left(f_{i_{2}}\right)\right\rangle_{G}+(-1)^{r-1}(r-1) ! \sum_{p^{\prime} \in \mathcal{P}_{i}^{(2)}} \prod_{i^{\prime} \in p^{\prime}}\left(f_{i_{1}^{\prime}}, A_{\text {mix }}^{ \pm} f_{i_{2}^{\prime}}\right)\right) .
$$

Moreover, it can be seen that the terms $\delta_{2, r}\left\langle Q\left(f_{i_{1}}\right) Q\left(f_{i_{2}}\right)\right\rangle_{G}$ can be ignored, because terms with at least one factor of the form $\delta_{2, r}\left\langle Q\left(f_{i_{1}}\right) Q\left(f_{i_{2}}\right)\right\rangle_{G}$ cannot contain $k$ factors of the $A$ type. Therefore, the following expression exactly sums up all the terms with $k$ factors of the $A$ type:

$$
-\sum_{p \in \mathcal{P}^{(2,4, \ldots, 2 k-2)}} \prod_{i \in p}(-1)^{r / 2-1}(r / 2-1) ! \sum_{p^{\prime} \in \mathcal{P}_{i}^{(2)}} \prod_{i^{\prime} \in p^{\prime}}\left(f_{i_{1}^{\prime}}, A_{\text {mix }}^{ \pm} f_{i_{2}^{\prime}}\right) \sim \sum_{p \in \mathcal{P}^{(2)}} \prod_{i \in p}\left(f_{i_{1}}, A_{\text {mix }}^{ \pm} f_{i_{2}}\right) .
$$

The similarity relation is straightforward to see, but all of these terms appear multiple times. We must approach this counting problem in a structural way. To do so, we translate our problem of set partitioning to an equivalent problem of integer partitioning. ${ }^{6}$ Specifically, in our derivation, the index set $\{1, \ldots, 2 k\}$ of which we have considered the set partitions can be linked to the integer

\footnotetext{
${ }^{6}$ An integer partition denotes a way of writing one integer as a sum of other integers. For example, $(2,4,6)$ would be an integer partition of $12=2+4+6$.
} 
partitions of the integer $k$ (because all the subsets in our partitions have an even number of elements). For example, when we think of the set partition $\{\{1,2\} ;\{3,4,5,6\}\}$, of an index set with $k=3$, we can associate it with the integer partition $(1,2)$ of the integer 3. However, also other set partitions are associated with the integer partition $(1,2)$, e.g., $\{\{3,6\} ;\{1,2,4,5\}\}$. These integer partitions thus represent a class of set partitions. The procedure (A2) to evaluate the truncated correlation functions breaks the subsets, e.g., $\{3,6\}$ and $\{1,2,4,5\}$ in the set partition $\{\{3,6\} ;\{1,2,4,5\}\}$, into pair partitions. It now follows that one single pair partition $p^{\prime} \in \mathcal{P}^{(2)}$ can be obtained several times within the class of a specific integer partition. For example, $\{\{1,2\} ;\{3,6\} ;\{4,5\}\}$ can be obtained both by breaking up $\{\{1,2\} ;\{3,4,5,6\}\}$, but also by breaking up $\{\{3,6\} ;\{1,2,4,5\}\}$ in pair partitions. However, in the end, it is the pair partition that determines which specific product of $A_{\text {mix }}^{ \pm}$matrix elements it obtained. In (A5), for our example, this implies that both $\{\{1,2\} ;\{3,4,5,6\}\}$ and $\{\{3,6\} ;\{1,2,4,5\}\}$ induce a term proportional to $\left(f_{1}, A_{\text {mix }}^{ \pm} f_{2}\right)\left(f_{3}, A_{\text {mix }}^{ \pm} f_{6}\right)\left(f_{4}, A_{\text {mix }}^{ \pm} f_{5}\right)$. The crucial point of grouping everything in classes of set partitions, associated with an integer partition, is that the factors $(-1)^{r / 2-1}(r / 2-1)$ ! are the same for any partition within the class. After all, the $r$ values indicated the number of elements in the subsets and therefore the $r / 2$ are the integers constituting the set partitions.

It is instructive to rewrite (A5) in terms of integer partitions

$$
\begin{aligned}
&- \sum_{p \in \mathcal{P}^{(2,4, \ldots, 2 k-2)}} \prod_{i \in p}(-1)^{r / 2-1}(r / 2-1) ! \sum_{p^{\prime} \in \mathcal{P}_{i}^{(2)}} \prod_{i^{\prime} \in p^{\prime}}\left(f_{i_{1}^{\prime}}, A_{\text {mix }}^{ \pm} f_{i_{2}^{\prime}}\right) \\
&=-\sum_{\substack{\left(I_{1}, \ldots, I_{q}\right) \\
I_{1}+\ldots+I_{q}=k \\
I_{1}, \ldots, I_{q}<k}}\left(\prod_{j=1}^{q}(-1)^{I_{j}-1}\left(I_{j}-1\right) !\right)\left(\sum_{p \in \mathcal{P}^{\left(2 I_{1}, \ldots, 2 I_{q}\right)}} \prod_{i \in p}\left\{\sum_{p^{\prime} \in \mathcal{P}_{i}^{(2)}} \prod_{i^{\prime} \in p^{\prime}}\left(f_{i_{1}^{\prime}}, A_{\mathrm{mix}}^{ \pm} f_{i_{2}^{\prime}}\right)\right\}\right),
\end{aligned}
$$

where $q$ is not fixed and depends on the integer partition. We already argued that the specific terms of the form $\prod_{i \in p}\left(f_{i_{1}}, A_{\text {mix }}^{ \pm} f_{i_{2}}\right)$ occur several times. What remains is to count their multiplicity for a given set partition.

Let us now focus on one specific integer partition $\left(I_{1}, \ldots, I_{q}\right)$. To count the multiplicity of a specific product of $A_{g}$ functions within this class of partitions, this product is associated with a specific pair partition $p^{\prime} \in \mathcal{P}^{(2)}$ and our counting process will consist of counting in how many partitions of the class $\left(I_{1}, \ldots, I_{q}\right)$ we can embed this specific pair partition. This problem is equivalent to asking in how many ways we can group $k$ elements in subsets of $I_{1}, I_{2}, \ldots$, and $I_{q}$ elements. Here these $k$ elements are the pairs in the pair partition. This combinatoric problem is solved using the multinomial coefficient such that we find

$$
\frac{k !}{\prod_{j=1}^{q} I_{q} !}
$$

An important additional ingredient is that this is independent of the specific pair partition we choose; they all occur with the same multiplicity within the specific integer partition $\left(I_{1}, \ldots, I_{q}\right)$. This implies that

$$
\sum_{p \in \mathcal{P}^{\left(2 I_{1}, \ldots, 2 I_{q}\right)}} \prod_{i \in p}\left\{\sum_{p^{\prime} \in \mathcal{P}_{i}^{(2)}} \prod_{i^{\prime} \in p^{\prime}}\left(f_{i_{1}^{\prime}}, A_{\mathrm{mix}}^{ \pm} f_{i_{2}^{\prime}}\right)\right\}=\frac{k !}{\prod_{j=1}^{q} I_{q} !} \sum_{p \in \mathcal{P}^{(2)}} \prod_{i \in p}\left(f_{i_{1}}, A_{\mathrm{mix}}^{ \pm} f_{i_{2}}\right) .
$$

If we now insert (A7) in (A6), we find

$$
\begin{aligned}
& -\sum_{p \in \mathcal{P}^{(2,4, \ldots, 2 k-2)}} \prod_{i \in p}(-1)^{r / 2-1}(r / 2-1) ! \sum_{p^{\prime} \in \mathcal{P}_{i}^{(2)}} \prod_{i^{\prime} \in p^{\prime}}\left(f_{i_{1}^{\prime}}, A_{\text {mix }}^{ \pm} f_{i_{2}^{\prime}}\right) . \\
& =-\sum_{\substack{\left(I_{1}, \ldots, I_{q}\right) \\
I_{1}+\cdots+I_{q}=k \\
I_{1}, \ldots, I_{q}<k}}\left(\prod_{j=1}^{q}(-1)^{I_{j}-1}\left(I_{j}-1\right) !\right)\left(\frac{k !}{\prod_{j=1}^{q} I_{q} !} \sum_{p \in \mathcal{P}^{(2)}} \prod_{i \in p}\left(f_{i_{1}}, A_{\text {mix }}^{ \pm} f_{i_{2}}\right)\right) \\
& =-\left(k ! \sum_{\substack{\left(I_{1}, \ldots, I_{q}\right) \\
I_{1}+\ldots+I_{q}=k \\
I_{1}, \ldots, I_{q}<k}} \prod_{j=1}^{q} \frac{(-1)^{I_{j}-1}}{I_{q}}\right) \sum_{p \in \mathcal{P}^{(2)}} \prod_{i \in p}\left(f_{i_{1}}, A_{\text {mix }}^{ \pm} f_{i_{2}}\right) .
\end{aligned}
$$

Finally, we use that

$$
\sum_{\substack{\left(I_{1}, \ldots, I_{q}\right) \\ I_{1}+\cdots+I_{q}=k \\ I_{1}, \ldots, I_{q} \leqslant k}} \prod_{j=1}^{q} \frac{(-1)^{I_{j}-1}}{I_{q}}=0,
$$


which explicitly includes the integer partition $(k)$ of $k$, which was excluded in the derivation of (A8). This implies that

$$
\sum_{\substack{\left(I_{1}, \ldots, I_{q}\right) \\ I_{1}+\cdots+I_{q}=k \\ I_{1}, \ldots, I_{q}<k}} \prod_{j=1}^{q} \frac{(-1)^{I_{j}-1}}{I_{q}}=-\frac{(-1)^{k-1}}{k} .
$$

Inserting (A10) in (A8) therefore results in

$$
-\sum_{p \in \mathcal{P}^{(2,4, \ldots, 2 k-2)}} \prod_{i \in p}(-1)^{r / 2-1}(r / 2-1) ! \sum_{p^{\prime} \in \mathcal{P}_{i}^{(2)}} \prod_{i^{\prime} \in p^{\prime}}\left(f_{i_{1}^{\prime}}, A_{\text {mix }}^{ \pm} f_{i_{2}^{\prime}}\right)=(-1)^{k-1}(k-1) ! \sum_{p \in \mathcal{P}^{(2)}} \prod_{i \in p}\left(f_{i_{1}}, A_{\text {mix }}^{ \pm} f_{i_{2}}\right),
$$

which is the final result for the contribution of the terms with only factors of the form $\left(f_{i_{1}}, A_{\text {mix }}^{ \pm} f_{i_{2}}\right)$.

It now remains to show that all other terms, in which factors of the form $\left.\left\langle Q\left(f_{i_{1}}\right) Q\left(f_{i_{2}}\right)\right)\right\rangle_{G}$ appear, vanish. We will use primarily the same apparatus to prove this and start by observing that only integer partitions with a component that is 1 can lead to a term $\left.\left\langle Q\left(f_{i_{1}}\right) Q\left(f_{i_{2}}\right)\right)\right\rangle_{G}$. Therefore, we focus on a class associated with the integer partition

$$
(\underbrace{1, \ldots, 1}_{x<k-1}, I_{1}, \ldots I_{q^{\prime}}) \quad \text { with } I_{1}+\cdots+I_{q^{\prime}}=k-x
$$

where the $x$ first terms are particularly associated with a factor $\left\langle Q\left(f_{i_{1}}\right) Q\left(f_{i_{2}}\right)\right\rangle_{G}$. We exclude the cases $x=k$ and $x=k-1$ because there $\operatorname{tr}\left\{\rho Q\left(f_{1}\right) \cdots Q\left(f_{2 k}\right)\right\}$ in (A1) must also be considered. We can group the terms with $x$ factors $\left\langle Q\left(f_{i_{1}}\right) Q\left(f_{i_{2}}\right)\right\rangle_{G}$ in (A4) as

$$
\begin{aligned}
& -\sum_{\substack{\left(1, \ldots, 1, I_{1}, \ldots, I_{q^{\prime}}\right) \\
I_{1}+\cdots+I_{q^{\prime}}=k-x \\
I_{1}, \ldots, I_{q^{\prime}} \leqslant k-x}} \sum_{\substack{\mathcal{X} \subset \mathcal{I} \\
\# \mathcal{X}=2 x}}\left(\sum_{p \in \mathcal{P}_{\mathcal{X}}^{(2)}} \prod_{i \in p}\left\langle Q\left(f_{i_{1}}\right) Q\left(f_{i_{2}}\right)\right\rangle_{G}\right)\left(\prod_{j=1}^{q^{\prime}}(-1)^{I_{j}-1}\left(I_{j}-1\right) !\right)\left(\sum_{j \in \mathcal{P}_{\mathcal{I} \backslash \mathcal{X}}^{\left(2 I_{1}, \ldots, 2 I_{q^{\prime}}\right)}} \prod_{i \in p}\left\{\sum_{\substack{p^{\prime} \in \mathcal{P}_{i}^{(2)} \\
i^{\prime} \in p^{\prime}}}\left(f_{i_{1}^{\prime}}, A_{\mathrm{mix}}^{ \pm} f_{i_{2}^{\prime}}\right)\right\}\right) \\
& =-\sum_{\substack{\mathcal{X} \subset \mathcal{I} \\
\# \mathcal{X}=2 x}}\left(\sum_{p \in \mathcal{P}_{\mathcal{X}}^{(2)}} \prod_{i \in p}\left\langle Q\left(f_{i_{1}}\right) Q\left(f_{i_{2}}\right)\right\rangle_{G}\right)\left(\sum_{\substack{\left(I_{1}, \ldots, I_{q^{\prime}}\right) \\
I_{1}+\cdots+I_{q}=k-x \\
I_{1}, \ldots, I_{q^{\prime}} \leqslant k-x}}\left(\prod_{j=1}^{q^{\prime}}(-1)^{I_{j}-1}\left(I_{j}-1\right) !\right)\right. \\
& \times\left(\sum_{p \in \mathcal{P}_{\mathcal{I} \backslash \mathcal{X}}^{\left(2 I_{1}, \ldots, 2 I_{q^{\prime}}\right)}} \prod_{i \in p}\left\{\sum_{p^{\prime} \in \mathcal{P}_{i}^{(2)}} \prod_{i^{\prime} \in p^{\prime}}\left(f_{i_{1}^{\prime}}, A_{\mathrm{mix}}^{ \pm} f_{i_{2}^{\prime}}\right)\right\}\right),
\end{aligned}
$$

where we introduce $\mathcal{I}=\{1,2, \ldots, 2 k\}$. The set $\mathcal{X}$ contains the subset of indices with which we associate the factors $\left\langle Q\left(f_{i_{1}}\right) Q\left(f_{i_{2}}\right)\right\rangle_{G}$; the other indices $\mathcal{I} \backslash \mathcal{X}$ have a factor of the $A$ type connected to them. We introduce the notation $\mathcal{P}_{\mathcal{X}}^{(2)}$ to denote the pair partitions of the set $\mathcal{X}$, whereas $\mathcal{P}_{\mathcal{I} \backslash \mathcal{X}}^{\left(2 I_{1}, \ldots, 2 I_{q^{\prime}}\right)}$ are all the possible even partitions of $\mathcal{I} \backslash \mathcal{X}$.

We can limit our efforts to understanding that

$$
\begin{aligned}
& \left.\sum_{\substack{\left(I_{1}, \ldots, I_{q^{\prime}}\right) \\
I_{1}+\cdots+I_{q^{\prime}}=k-x \\
I_{1}, \ldots, I_{q^{\prime}} \leqslant k-x}}\left(\prod_{j=1}^{q^{\prime}}(-1)^{I_{j}-1}\left(I_{j}-1\right) !\right)\left(\sum_{\substack{\left(2 I_{1}, \ldots, 2 I_{q^{\prime}}\right) \\
p \in \mathcal{P}_{\mathcal{I} \backslash \mathcal{X}}}} \prod_{i \in p} \sum_{p^{\prime} \in \mathcal{P}_{i}^{(2)}} \prod_{i^{\prime} \in p^{\prime}}\left(f_{i_{1}^{\prime}}, A_{\text {mix }}^{ \pm} f_{i_{2}^{\prime}}\right)\right\}\right) \\
= & \sum_{\substack{\left(I_{1}, \ldots, I_{q^{\prime}}\right) \\
I_{1}+\cdots+I_{q^{\prime}}=k-x \\
I_{1}, \ldots, I_{q^{\prime}} \leqslant k-x}}\left(\prod_{j=1}^{q^{\prime}}(-1)^{I_{j}-1}\left(I_{j}-1\right) !\right)\left(\frac{(k-x) !}{\prod_{j=1}^{q^{\prime}} I_{j} !} \sum_{p \in \mathcal{P}_{\mathcal{I} \backslash \mathcal{X}}^{(2)}} \prod_{i \in p}\left(f_{i_{1}}, A_{\text {mix }}^{ \pm} f_{i_{2}}\right)\right)
\end{aligned}
$$




$$
\begin{aligned}
& =\left(\sum_{\substack{\left(I_{1}, \ldots, I_{q^{\prime}}\right) \\
I_{1}+\cdots+I_{q^{\prime}}=k-x \\
I_{1}, \ldots, I_{q^{\prime}} \leqslant k-x}} \prod_{j=1}^{q^{\prime}}(-1)^{I_{j}-1} \frac{(k-x) !}{I_{j}}\right) \sum_{p \in \mathcal{P}_{\mathcal{T} \backslash \mathcal{X}}^{(2)}} \prod_{i \in p}\left(f_{i_{1}}, A_{\text {mix }}^{ \pm} f_{i_{2}}\right) \\
& =0 .
\end{aligned}
$$

The above steps use exactly the same reasoning as the derivation of (A8). Finally, we used (A9) to obtain that the expression is zero.

The case where $x=k$ can be treated explicitly. This contribution reads

$$
\left\langle Q\left(f_{1}\right) \cdots Q\left(f_{2 k}\right)\right\rangle_{G}-\sum_{p \in \mathcal{P}^{(2)}} \prod_{i \in p}\left\langle Q\left(f_{i_{1}}\right) Q\left(f_{i_{2}}\right)\right\rangle_{G}=0,
$$

because it is the truncated correlation function in a Gaussian state. The case $x=k-1$, i.e., where there is exactly one factor of the form $A_{g}$, leads to a contribution

$$
\begin{aligned}
& \sum_{p \in \mathcal{P}^{(2)}} \sum_{i \in p}\left(\left(f_{i_{1}}, A_{\text {mix }}^{ \pm} f_{i_{2}}\right) \prod_{j \in p \backslash i}\left\langle Q\left(f_{j_{1}}\right) Q\left(f_{j_{2}}\right)\right\rangle_{G}\right)
\end{aligned}
$$

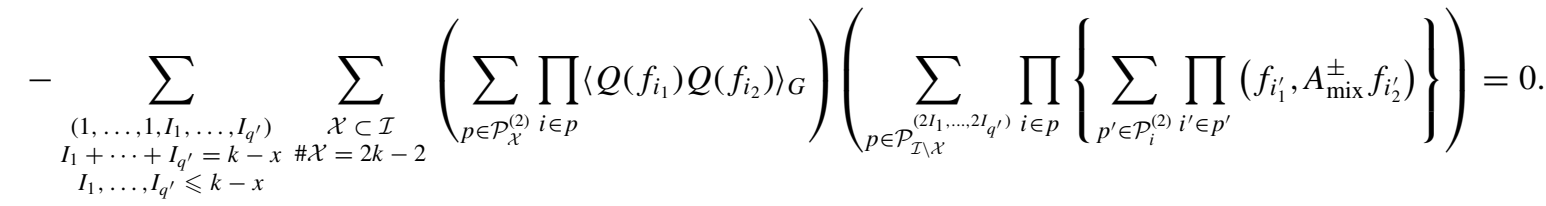

This contribution vanishes because in this case $\mathcal{I} \backslash \mathcal{X}=\left\{i_{1}, i_{2}\right\}$. Therefore, the second sum is equivalent to the first.

We have now evaluated all the different terms in (A1), assuming that for lower orders (A2) holds. Indeed, for our final result, we have

$$
\begin{aligned}
\left\langle Q\left(f_{1}\right) \ldots Q\left(f_{2 k}\right)\right\rangle_{T} & =(A 11)+(A 13)+(A 14)+(A 15) \\
& =(-1)^{k-1}(k-1) ! \sum_{p \in \mathcal{P}^{(2)}} \prod_{i \in p}\left(f_{i_{1}}, A_{\text {mix }}^{ \pm} f_{i_{2}}\right) \quad \text { for } k>1 .
\end{aligned}
$$

This concludes the derivation of (44).

\section{APPENDIX B: COMPUTATION OF THE WIGNER FUNCTION (50)}

We treat the problem explicitly in the basis of eigenvectors $\mathcal{E}=\left\{e_{1}, \ldots, e_{2 m}\right\}$ of $V$. Note that these eigenvectors in general do not respect the symplectic structure of phase space. The following steps are merely technical tricks to evaluate the Fourier transform and cannot be directly connected to a well-defined mode space. In (49) we may write

$$
\alpha=\sum_{j=1}^{2 m} \alpha_{j} e_{j}, \quad \beta=\sum_{j=1}^{2 m} \beta_{j} e_{j} .
$$

We start from (B1), where it now follows that

$$
\left(\alpha, A_{\mathrm{mix}}^{ \pm} \alpha\right)=\sum_{i, j=1}^{m} \alpha_{i} \alpha_{j}\left(e_{i}, A_{\mathrm{mix}}^{ \pm} e_{j}\right) .
$$

Upon inserting (B2) in (49) and using the linearity of the integration we find explicitly

$$
\begin{aligned}
W\left(\beta_{1}, \ldots, \beta_{2 m}\right)= & \prod_{k=1}^{2 m} \frac{1}{2 \pi} \int_{-\infty}^{\infty} d \alpha_{k} \exp \left\{-\frac{v_{k}\left(\alpha_{k}\right)^{2}}{2}-i \alpha_{k} \beta_{k}\right\}-\sum_{j=1}^{2 m} \frac{\left(e_{j}, A_{\mathrm{mix}}^{ \pm} e_{j}\right)}{8 \pi^{2}} \int_{-\infty}^{\infty} d \alpha_{j}\left(\alpha_{j}\right)^{2} \exp \left\{-\frac{v_{j}\left(\alpha_{j}\right)^{2}}{2}-i \alpha_{j} \beta_{j}\right\} \\
& \times \prod_{\substack{k=1 \\
k \neq j}}^{\infty m} \frac{1}{2 \pi} \int_{-\infty}^{\infty} d \alpha_{q}^{(j)} \exp \left\{-\frac{v_{q}^{(j)}\left(\alpha_{q}^{(j)}\right)^{2}}{2}-i \alpha_{q}^{(j)} \beta_{q}^{(j)}\right\}-\sum_{\substack{j, j^{\prime}=1 \\
j \neq j^{\prime}}}^{2 m} \frac{\left(e_{j}, A_{\mathrm{mix}}^{ \pm} e_{j^{\prime}}\right)}{8 \pi^{2}} \int_{-\infty}^{\infty} d \alpha_{j} d \alpha_{j^{\prime}} \alpha_{j} \alpha_{j^{\prime}}
\end{aligned}
$$




$$
\begin{aligned}
& \times \exp \left\{-\frac{v_{j}\left(\alpha_{j}\right)^{2}}{2}-\frac{v_{j^{\prime}}\left(\alpha_{j^{\prime}}\right)^{2}}{2}-i \alpha_{j} \beta_{j}-i \alpha_{j^{\prime}} \beta_{j^{\prime}}\right\} \prod_{\substack{k=1 \\
k \neq j, k \neq j^{\prime}}}^{2 m} \frac{1}{2 \pi} \int_{-\infty}^{\infty} d \alpha_{k} \exp \left\{-\frac{v_{k}\left(\alpha_{k}\right)^{2}}{2}-i \alpha_{k} \beta_{k}\right\} \\
= & \frac{1}{(2 \pi)^{m}}\left(1+\sum_{j=1}^{m} \frac{\left(e_{j}, A_{\mathrm{mix}}^{ \pm} e_{j}\right)\left[\left(\beta_{j}\right)^{2}-v_{j}\right]}{2\left(v_{j}\right)^{2}}+\sum_{\substack{j, j^{\prime}=1 \\
j \neq j^{\prime}}}^{2 m} \frac{\left(e_{j}, A_{\text {mix }}^{ \pm} e_{j^{\prime}}\right) \beta_{j} \beta_{j^{\prime}}}{2 v_{j} v_{j^{\prime}}}\right) \prod_{j=1}^{2 m} \frac{1}{\sqrt{v_{j}}} \exp \left\{-\frac{\left(\beta_{j}\right)^{2}}{2 v_{j}}\right\} .
\end{aligned}
$$

The last step consists simply of evaluating the Fourier transforms and grouping the terms. It must be stressed that, since this basis is not symplectic, we cannot interpret the Wigner function in this form as a quasiprobability distribution on phase space. Therefore, we require a basis-independent way of representing the function. This can be obtained by regrouping the terms and observing that

$$
\begin{aligned}
& \sum_{j=1}^{m} \frac{\left(e_{j}, A_{\mathrm{mix}}^{ \pm} e_{j}\right)\left(\beta_{j}\right)^{2}}{2\left(v_{j}\right)^{2}}+\sum_{\substack{j, j^{\prime}=1 \\
j \neq j^{\prime}}}^{2 m} \frac{\left(e_{j}, A_{\mathrm{mix}}^{ \pm} e_{j^{\prime}}\right) \beta_{j} \beta_{j^{\prime}}}{2 v_{j} v_{j^{\prime}}} \\
& \quad=\sum_{j, j^{\prime}=1}^{2 m} \frac{\left(e_{j}, A_{\mathrm{mix}}^{ \pm} e_{j^{\prime}}\right) \beta_{j} \beta_{j^{\prime}}}{2 v_{j} v_{j^{\prime}}}=\frac{1}{2} \sum_{j, j^{\prime}=1}^{2 m} \beta_{j} \beta_{j^{\prime}}\left(e_{j}, V^{-1} A_{\mathrm{mix}}^{ \pm} V^{-1} e_{j^{\prime}}\right) \\
& \quad=\frac{1}{2}\left(\sum_{j} \beta_{j} e_{j}, V^{-1} A_{\mathrm{mix}}^{ \pm} V^{-1} \sum_{j^{\prime}} \beta_{j^{\prime}} e_{j^{\prime}}\right)=\frac{1}{2}\left(\beta, V^{-1} A_{\text {mix }}^{ \pm} V^{-1} \beta\right),
\end{aligned}
$$

where we use the linearity of the inner product and that $V e_{j}=v_{j} e_{j}$. Similarly, we obtain

$$
\begin{aligned}
& -\sum_{j=1}^{m} \frac{\left(\beta_{j}\right)^{2}}{2 v_{j}}=-\frac{\left(\beta, V^{-1} \beta\right)}{2}, \\
& -\sum_{j=1}^{m} \frac{\left(e_{j}, A_{\text {mix }}^{ \pm} e_{j}\right)}{2 v_{j}}=\frac{1}{2} \operatorname{tr}\left\{V^{-1} A_{\text {mix }}^{ \pm}\right\}
\end{aligned}
$$

such that ultimately

$$
W(\beta)=\frac{1}{2^{m+1} \pi^{m} \sqrt{\operatorname{det} V}}\left[\left(\beta, V^{-1} A_{\text {mix }}^{ \pm} V^{-1} \beta\right)-\operatorname{tr}\left(V^{-1} A_{\text {mix }}^{ \pm}\right)+2\right] e^{-\left(\beta, V^{-1} \beta\right) / 2} .
$$

\section{APPENDIX C: PHOTON ADDITION AND SUBTRACTION WITH DISPLACED STATES}

The discussion in Sec. III B 3 requires an expression for the Wigner function for a displaced squeezed vacuum from (to) which a photon is subtracted (added). In this appendix we go one step beyond this need and we derive the Wigner function for photon subtraction or addition in a general displaced Gaussian state that need not be pure. Our derivation exploits the previously obtained result (50) for the nondisplaced case.

Any displaced Gaussian state can be written as

$$
\rho_{\xi}=D(\xi) \rho_{G} D(-\xi)
$$

where $\rho_{G}$ is a nondisplaced Gaussian state, characterized by a covariance matrix $V$. A photon-subtracted state can then be written as

$$
\rho=\frac{a(g) D(\xi) \rho_{G} D(-\xi) a^{\dagger}(g)}{\langle D(-\xi) \hat{n}(g) D(\xi)\rangle_{G}},
$$

with $\hat{n}(g)$ the number operators in mode $g$. It is not hard to evaluate that

$$
\langle D(-\xi) \hat{n}(g) D(\xi)\rangle_{G}=\langle\hat{n}(g)\rangle_{G}+\frac{1}{4}\left[(\xi, g)^{2}+(\xi, J g)^{2}\right] .
$$

We may now use that

$$
\begin{aligned}
D(\xi) a(g) & =a(g) D(\xi)-\frac{1}{2}(\xi,[\mathbb{1}+i J] g) D(\xi), \\
D(\xi) a^{\dagger}(g) & =a^{\dagger}(g) D(\xi)-\frac{1}{2}(\xi,[\mathbb{1}-i J] g) D(\xi)
\end{aligned}
$$




$$
\begin{aligned}
= & \frac{W_{G}(\beta-\xi)}{2\langle\hat{n}(g)\rangle_{G}+\frac{1}{2}\left[(\xi, g)^{2}+(\xi, J g)^{2}\right]}\left(\langle\hat{n}(g)\rangle_{G}\left\{\left([\beta-\xi], V^{-1} A_{g}^{-} V^{-1}[\beta-\xi]\right)-\operatorname{tr}\left(V^{-1} A_{\mathrm{g}}^{-}\right)+2\right\}\right. \\
& \left.+\frac{1}{2}\left[(\xi, g)^{2}+(\xi, J g)^{2}\right]+\left(\xi,\left[P_{g}+P_{J g}\right]\left[\mathbb{1}-V^{-1}\right][\beta-\xi]\right)\right),
\end{aligned}
$$

which is the final Wigner function for photon subtraction from a displaced state.

A completely analogous calculation can be performed for photon addition. Here the starting point is

$$
\rho=\frac{a^{\dagger}(g) D(\xi) \rho_{G} D(-\xi) a(g)}{\langle D(-\xi) \hat{n}(g) D(\xi)\rangle_{G}+1} .
$$

Going through the same steps of calculation, while taking into account the changes in signs, leads us to

$$
\begin{aligned}
\chi(\alpha)= & \frac{e^{i(\xi, \alpha)}}{\langle\hat{n}(g)\rangle_{G}+1+\frac{1}{4}\left[(\xi, g)^{2}+(\xi, J g)^{2}\right]}\left(\left[\langle\hat{n}(g)\rangle_{G}+1\right] \chi_{+}(\alpha)+\frac{1}{4}\left[(\xi, g)^{2}+(\xi, J g)^{2}\right] \chi_{G}(\alpha)\right. \\
& \left.+\frac{1}{2}\left(\xi,\left\{\left[P_{J g}+P_{g}\right][J+i \mathbb{1}]-\left[P_{g}+P_{J g}\right] J+i\left[P_{g}+P_{J g}\right] V\right\} \alpha\right) \chi_{G}(\alpha)\right) .
\end{aligned}
$$

This directly leads to a Wigner function of the form

$$
\begin{aligned}
W_{\xi}^{+}(\beta)= & \frac{W_{G}(\beta-\xi)}{\langle\hat{n}(g)\rangle_{G}+1+\frac{1}{4}\left[(\xi, g)^{2}+(\xi, J g)^{2}\right]}\left(\frac{\langle\hat{n}(g)\rangle_{G}+1}{2}\left\{\left([\beta-\xi], V^{-1} A_{g}^{+} V^{-1}[\beta-\xi]\right)-\operatorname{tr}\left(V^{-1} A_{g}^{+}\right)+2\right\}\right. \\
& \left.\left.+\frac{1}{4}\left[(\xi, g)^{2}+(\xi, J g)^{2}\right)\right]+\frac{1}{2}\left(\xi,\left[P_{g}+P_{J g}\right]\left[\mathbb{1}+V^{-1}\right][\beta-\xi]\right)\right) .
\end{aligned}
$$

With a little more rewriting, we find

$$
\begin{aligned}
W_{\xi}^{ \pm}(\beta)= & \frac{W_{G}(\beta-\xi)}{\operatorname{tr}\left[\left(V+\|\xi\|^{2} P_{\xi} \pm \mathbb{1}\right)\left(P_{g}+P_{J g}\right)\right]}\left\{\left\|\left(P_{g}+P_{J g}\right)\left(\mathbb{1} \pm V^{-1}\right)(\beta-\xi)\right\|^{2}\right. \\
& \left.+2\left(\xi,\left[P_{g}+P_{J g}\right]\left[\mathbb{1} \pm V^{-1}\right][\beta-\xi]\right)+\operatorname{tr}\left[\left(P_{g}+P_{J g}\right)\left(\|\xi\|^{2} P_{\xi}-V^{-1} \mp \mathbb{1}\right)\right]\right\} .
\end{aligned}
$$

[1] M. Chen, N. C. Menicucci, and O. Pfister, Phys. Rev. Lett. 112, 120505 (2014)

[2] J.-i. Yoshikawa, S. Yokoyama, T. Kaji, C. Sornphiphatphong, Y. Shiozawa, K. Makino, and A. Furusawa, APL Photon. 1, 060801 (2016).

[3] S. Rahimi-Keshari, T. C. Ralph, and C. M. Caves, Phys. Rev. X 6, 021039 (2016).

[4] N. C. Menicucci, Phys. Rev. Lett. 112, 120504 (2014).

[5] F. Arzani, N. Treps, and G. Ferrini, Phys. Rev. A 95, 052352 (2017).

[6] V. Parigi, A. Zavatta, M. Kim, and M. Bellini, Science 317, 1890 (2007).

[7] A. Ourjoumtsev, A. Dantan, R. Tualle-Brouri, and P. Grangier, Phys. Rev. Lett. 98, 030502 (2007).

[8] H. Takahashi, J. S. Neergaard-Nielsen, M. Takeuchi, M. Takeoka, K. Hayasaka, A. Furusawa, and M. Sasaki, Nat. Photon. 4, 178 (2010).

[9] C. Navarrete-Benlloch, R. García-Patrón, J. H. Shapiro, and N. J. Cerf, Phys. Rev. A 86, 012328 (2012).

[10] M. Dakna, T. Anhut, T. Opatrný, L. Knöll, and D.-G. Welsch, Phys. Rev. A 55, 3184 (1997).

[11] V. A. Averchenko, V. Thiel, and N. Treps, Phys. Rev. A 89, 063808 (2014).

[12] V. Averchenko, C. Jacquard, V. Thiel, C. Fabre, and N. Treps, New J. Phys. 18, 083042 (2016).

[13] Y.-S. Ra, C. Jacquard, A. Dufour, C. Fabre, and N. Treps, Phys. Rev. X 7, 031012 (2017).
[14] S. L. Braunstein and P. van Loock, Rev. Mod. Phys. 77, 513 (2005).

[15] C. Weedbrook, S. Pirandola, R. García-Patrón, N. J. Cerf, T. C. Ralph, J. H. Shapiro, and S. Lloyd, Rev. Mod. Phys. 84, 621 (2012).

[16] G. S. Agarwal and K. Tara, Phys. Rev. A 43, 492 (1991).

[17] M. S. Kim, E. Park, P. L. Knight, and H. Jeong, Phys. Rev. A 71, 043805 (2005).

[18] A. Biswas and G. S. Agarwal, Phys. Rev. A 75, 032104 (2007).

[19] M. S. Kim, J. Phys. B 41, 133001 (2008).

[20] D. W. Robinson, Commun. Math. Phys. 1, 159 (1965).

[21] D. Petz, An Invitation to the Algebra of Canonical Commutation Relations, Leuven Notes in Mathematical and Theoretical Physics Series A (Leuven University Press, Leuven, 1990), Vol. 2.

[22] O. Bratteli and D. W. Robinson, Operator Algebras and Quantum Statistical Mechanics: Equilibrium States. Models in Quantum Statistical Mechanics, Theoretical and Mathematical Physics (Springer, Berlin, 1997).

[23] A. Verbeure, Many-Body Boson Systems: Half a Century Later, Theoretical and Mathematical Physics (Springer, New York, 2011).

[24] R. Hudson, Rep. Math. Phys. 6, 249 (1974).

[25] F. Soto and P. Claverie, J. Math. Phys. 24, 97 (1983).

[26] A. Mandilara, E. Karpov, and N. J. Cerf, Phys. Rev. A 79, 062302 (2009). 
[27] A. Kenfack and K. Życzkowski, J. Opt. B 6, 396 (2004).

[28] A. Mari and J. Eisert, Phys. Rev. Lett. 109, 230503 (2012).

[29] M. Walschaers, C. Fabre, V. Parigi, and N. Treps, Phys. Rev. Lett. 119, 183601 (2017).

[30] N. Treps, V. Delaubert, A. Maître, J. M. Courty, and C. Fabre, Phys. Rev. A 71, 013820 (2005).

[31] S. Jiang, N. Treps, and C. Fabre, New J. Phys. 14, 043006 (2012).

[32] P. J. Mosley, J. S. Lundeen, B. J. Smith, P. Wasylczyk, A. B. U'Ren, C. Silberhorn, and I. A. Walmsley, Phys. Rev. Lett. 100, 133601 (2008).

[33] J. von Neumann, Math. Ann. 104, 570 (1931).

[34] M. Beck, Phys. Rev. Lett. 84, 5748 (2000).

[35] S. Armstrong, J.-F. Morizur, J. Janousek, B. Hage, N. Treps, P. K. Lam, and H.-A. Bachor, Nat. Commun. 3, 1026 (2012).

[36] Y. Cai, J. Roslund, G. Ferrini, F. Arzani, X. Xu, C. Fabre, and N. Treps, Nat. Commun. 8, 15645 (2017).

[37] D. W. Robinson, Commun. Math. Phys. 1, 89 (1965).

[38] S. Gerke, J. Sperling, W. Vogel, Y. Cai, J. Roslund, N. Treps, and C. Fabre, Phys. Rev. Lett. 114, 050501 (2015).
[39] M. M. Wolf, G. Giedke, O. Krüger, R. F. Werner, and J. I. Cirac, Phys. Rev. A 69, 052320 (2004).

[40] J. Eisert and M. M. Wolf, in Quantum Information with Continuous Variables of Atoms and Light, edited by N. J. Cerf, G. Leuchs, and E. S. Polzik (Imperial College Press, London, 2007).

[41] A. Zavatta, V. Parigi, and M. Bellini, Phys. Rev. A 75, 052106 (2007).

[42] R. J. Glauber, Phys. Rev. 130, 2529 (1963).

[43] T. Mansour, Combinatorics of Set Partitions (CRC Press, Boca Raton, 2013).

[44] G. S. Agarwal and A. Biswas, New J. Phys. 7, 211 (2005).

[45] A. A. Valido, F. Levi, and F. Mintert, Phys. Rev. A 90, 052321 (2014).

[46] T. Das, R. Prabhu, A. Sen(De), and U. Sen, Phys. Rev. A 93, 052313 (2016).

[47] M. Gu, C. Weedbrook, N. C. Menicucci, T. C. Ralph, and P. van Loock, Phys. Rev. A 79, 062318 (2009).

[48] M. M. Wolf, G. Giedke, and J. I. Cirac, Phys. Rev. Lett. 96, 080502 (2006). 